Research Article

\title{
The Effect of Uncertainty in the Excitation on the Vibration Input Power to a Structure
}

\author{
A. Putra ${ }^{1}$ and B. R. Mace ${ }^{2}$ \\ ${ }^{1}$ Faculty of Mechanical Engineering, Universiti Teknikal Malaysia Melaka, Hang Tuah Jaya, 76100 Durian Tunggal, Melaka, Malaysia \\ ${ }^{2}$ Department of Mechanical Engineering, University of Auckland, Auckland 1142, New Zealand
}

Correspondence should be addressed to A. Putra; azma.putra@utem.edu.my

Received 30 April 2013; Accepted 18 July 2013

Academic Editor: Marc Thomas

Copyright (C) 2013 A. Putra and B. R. Mace. This is an open access article distributed under the Creative Commons Attribution License, which permits unrestricted use, distribution, and reproduction in any medium, provided the original work is properly cited.

In structural dynamic systems, there is inevitable uncertainty in the input power from a source to a receiver. Apart from the nondeterministic properties of the source and receiver, there is also uncertainty in the excitation. This comes from the uncertainty of the forcing location on the receiver and, for multiple contact points, the relative phases, the force amplitude distribution at those points, and also their spatial separation. This paper investigates quantification of the uncertainty using possibilistic or probabilistic approaches. These provide the maximum and minimum bounds and the statistics of the input power, respectively. Expressions for the bounds, mean, and variance are presented. First the input power from multiple point forces acting on an infinite plate is examined. The problem is then extended to the input power to a finite plate described in terms of its modes. The uncertainty due to the force amplitude is also discussed. Finally, the contribution of moment excitation to the input power, which is often ignored in the calculation, is investigated. For all cases, frequency band-averaged results are presented.

\section{Introduction}

The treatment of structure-borne sound sources remains a challenging problem. Structural excitation to a building floor, for example, by active components like pumps, compressors, fans, and motors, is an important mechanism of sound generation. To obtain an accurate prediction of the injected input power from such sources, both the source and the receiver must firstly be characterised. However in practical application, the variability of source and receiver properties including the lack of knowledge in the excitation force creates uncertainty in the input power. The problem is exacerbated because in practice there will usually be multiple contact points (typically four) and 6 degrees of freedom ( 3 for translation and 3 for rotation) at each, and that force and moment components at each contact point will contribute to the total input power. Therefore to assess the uncertainty, some quantification of the bounds, mean, and variance of the input power is of interest.

The uncertainty in vibrational energy due to random properties, for example, dimensions, shapes, boundary conditions, and so on in a simple receiver structure, such as a plate, has been described by Langley and Brown $[1,2]$, where expressions for the mean vibrational energy and its variance were developed. A closed form solution was presented for the relative variance as a function of modal overlap factor and the nature of the excitation [1]. In [2], the analysis was extended to the ensemble average of the frequency bandaveraged energy as a function of the frequency bandwidth. The analysis proceeded on the assumption that the natural frequencies form a random point process with statistics governed by, for example, the Gaussian orthogonal ensemble (GOE). The same type of analysis for a more complex system has also been proposed [3].

Regardless of the receiving structure, the concept of source descriptor has been proposed to characterise a source based on its ability to deliver power without necessarily knowing any information about the receiver $[4,5]$. Here, the concept of effective mobility $[6,7]$, that is, the ratio of the actual velocity at a point and in one direction, to the contributions of the excitations from all components and points, was used. 
The effective mobility concept was also employed in [8] to estimate the total power to an infinite beam through four contact points. The importance of having knowledge of the force distribution at the contact points was acknowledged. For this purpose, three simple force ratio assumptions were introduced, and the effects of force position, type of excitation, the structural loss factor, the receiver, and the number of contact points were investigated. It was found that the estimation is accurate only in the mass-controlled region and when the system approximated a symmetrical response. In a later paper [9], the force ratio was defined in terms of its statistical distribution. Simple expressions of the distribution at the mass-, stiffness-, and resonance-controlled regions were derived.

Characterisation of a source in practice can be approached by using a reception plate method $[10,11]$ which is based on laboratory measurements, where from measured mobilities and surface mean-square velocities of the receiver, the free velocities and mobilities of the source can be extracted.

This paper focuses only on the uncertainty in the excitation with the source and receiver assumed to be deterministic. The source may have multiple contact points. The uncertainty in input power due to the excitation phase, its location, and separation of the contact points is investigated. First some general comments are made. Broadband excitation is described, although only time-harmonic excitation is considered here with frequency averages subsequently being taken. The input power from multiple point forces to an infinite plate is examined to give an insight into the physical mechanisms involved. In practice, the receiver will have modes, although the modal overlap might be high. The input power to a finite plate is then analysed, where now the forcing location on the receiver becomes important. The mean and the variance of the input power averaged over force positions are investigated. The results are also presented in frequency-band averages.

The uncertainty in the input power due to uncertainty in the force amplitude for multiple contact points is also discussed for a simple case of two contact points. Rather than dealing with force ratios between contact points, the sum of the squared magnitudes of the forces is assumed known.

Finally, the inclusion of moments in the excitation is investigated and predictions of its contribution to the input power are made.

\section{Input Power and Uncertainty Assessment}

2.1. Time-Harmonic Excitation. Consider a vibrating source connected through a single or $N$ contact points to a receiver. For a time-harmonic excitation at frequency $\omega$, the input power is expressed as a function of mobility (or impedance) of the source and receiver $[10,12]$. This requires knowledge of both source and receiver mobilities and the so-called blocked force or free velocity of the source. In general, the mobilities are matrices and the blocked forces or the free velocities are vectors, with the elements relating to the various translational and rotational degrees of freedoms (DOFs) at the contact points. In this paper, however, the analysis is made by assuming that the force excitation is known, and the source mobility is assumed to be much smaller than that of the receiver, as is usually the case in practice. The input power is therefore given by

$$
P_{\text {in }}=\frac{1}{2} \operatorname{Re}\left\{\widetilde{\mathbf{F}}^{*} \widetilde{\mathbf{Y}} \widetilde{\mathbf{F}}\right\},
$$

where $\widetilde{\mathbf{F}}=\left[\begin{array}{ll}F_{1} e^{j \phi_{1}} & F_{2} e^{j \phi_{2}} \cdots F_{N} e^{j \phi_{N}}\end{array}\right]^{T}$ is the vector of the complex amplitudes of the time-harmonic forces and where * denotes the conjugate transpose. The $i$ th force has a real magnitude $F_{i}$ and phase $\phi_{i}$. The mobilities of the receiver are represented by an $N \times N$ matrix $\tilde{\mathbf{Y}}$. In this section, only forces are considered. Moment excitation is discussed in Section 6.

2.2. Broadband Excitation. For a broadband excitation over a frequency band $B$, the input power should be defined in terms of power spectral density and can be written as

$$
S_{P_{\mathrm{in}}}=\sum_{i}^{N} S_{i i} \operatorname{Re}\left\{\widetilde{Y}_{i i}\right\}+\sum_{\substack{i, k \\ i \neq k}}^{N} \operatorname{Re}\left\{\widetilde{S}_{i k}\right\} \operatorname{Re}\left\{\widetilde{Y}_{i k}\right\},
$$

where $S_{i i}$ and $\widetilde{S}_{i k}$ are the autospectral density and crossspectral density of the forces, respectively, and $\widetilde{Y}_{i k}$ is the transfer mobility between points $i$ and $k$. The cross-spectral densities would often in practice be difficult to measure. However, the coherence relates the auto- and cross-spectral densities of the excitation. Thus by assuming that only the autospectra of the forces are known, the cross-spectra are such that

$$
\left|\widetilde{S}_{i k}\right|= \pm \sqrt{\gamma^{2} S_{i i} S_{k k}}
$$

where $0 \leq \gamma^{2} \leq 1$ is the coherence. This gives maximum and minimum bounds to the magnitude of the cross-spectral density. A rather similar approach is proposed by Evans and Moorhouse [13] for the case of a rigid body source, where the real part of the cross-spectral density is predicted using the available data of the autospectra and the calculated free velocities at the contact points from the source rigid body modes. Hence, this is limited only to the mass-controlled region of the source at very low frequencies. The comparison with measured data shows a good agreement. However, neither of these approaches are implemented in this paper. All forces are assumed to be time harmonic.

2.3. Uncertainty Quantification. Two approaches are employed to describe the uncertainty in the input power, namely, possibilistic and probabilistic approaches [14]. The possibilistic approach gives an interval description of the input power, which lies between lower and upper bounds; that is,

$$
P_{\text {in }} \in\left[\underline{P_{\text {in }}} \overline{P_{\text {in }}}\right],
$$

where $P_{\text {in }}$ and $\overline{P_{\text {in }}}$ are the minimum and maximum bounds and $P_{\text {in }}$ is the interval variable. One example has been given in Section 2.2, where the input power can be bounded by using the spectral coherence data. 
The probabilistic approach gives information about the likelihood and probability of the input power. The variation is specified by a probability density function $\Pi$. If $\Pi(z)$ is a continuous function of some variable $z$, the mean or the expected value of the input power and its variance are defined by

$$
\begin{aligned}
& \mu_{P_{\text {in }}}=E\left[P_{\text {in }}\right]=\int_{z} P_{\text {in }}(z) \Pi(z) \mathrm{d} z, \\
& \sigma_{P_{\text {in }}}^{2}=\int_{z} P_{\text {in }}^{2}(z) \Pi(z) \mathrm{d} z-\left(\mu_{P_{\text {in }}}\right)^{2} .
\end{aligned}
$$

\section{An Infinite Plate Receiver}

The input power to an infinite plate, as an ideal simple structure, is first investigated. The point and transfer mobilities for an infinite plate are given by [15]

$$
\begin{aligned}
& \widetilde{Y}_{p}=\frac{1}{8 \sqrt{B_{e} m}}, \\
& \tilde{Y}_{t}=\widetilde{Y}_{p}\left[H_{0}^{(2)}(k L)-\frac{2 j}{\pi} K_{0}(k L)\right],
\end{aligned}
$$

where $H_{0}^{(2)}$ is the zeroth-order Hankel function of the second kind, $K_{0}$ is the zeroth-order modified Bessel function of the second kind, $m$ is the mass per unit area, and $B_{e}=E h^{3} / 12(1-$ $\left.\nu^{2}\right)$ is the bending stiffness of the plate having Young's modulus $E$, thickness $h$, and Poisson's ratio $v$. The input point mobility is purely real and independent of frequency, behaving as a damper. In the transfer mobility, the first and second terms represent propagating and near-field outgoing waves, respectively.

Assume an infinite plate is excited by two point forces separated by a distance $L$. From (1), the force vector is $\mathbf{F}=$ $\left\{F_{1} e^{j \phi_{1}} \quad F_{2} e^{j \phi_{2}}\right\}^{T}$ and the mobility is a $2 \times 2$ matrix. The total power is, thus, the sum of the input power at each location which yields

$$
P_{\text {in }}=\frac{1}{2} \operatorname{Re}\left\{\widetilde{Y}_{p}\right\}\left(F_{1}^{2}+F_{2}^{2}\right)+\operatorname{Re}\left\{\tilde{Y}_{t}\right\} F_{1} F_{2} \cos \varphi,
$$

where $\varphi=\phi_{1}-\phi_{2}$ is the phase difference between the two forces. Note that $\widetilde{Y}_{12}=\widetilde{Y}_{21}=\widetilde{Y}_{t}$. For the case of an infinite structure, the input mobility is the same at any position which implies that $\widetilde{Y}_{11}=\widetilde{Y}_{22}=\widetilde{Y}_{p}$.

3.1. Dependence on the Contact Points Separation. When the structural wavelength $\lambda$ is much larger than the separation of the excitation points $(\lambda \gg L)$, the transfer mobility is approximately equal to the point mobility. Therefore, $\widetilde{Y}_{p} \approx \widetilde{Y}_{t}$. For simplicity, if $F_{1}=F_{2}=F$, the input power becomes

$$
P_{\text {in }}=\operatorname{Re}\left\{\widetilde{Y}_{p}\right\} F^{2}(1+\cos \varphi) .
$$

From (8), the maximum and minimum input power are

$$
\begin{gathered}
\overline{P_{\text {in }}}=2 F^{2} \operatorname{Re}\left\{\tilde{Y}_{p}\right\}, \quad \text { for } \varphi=2 n \pi \\
\underline{P_{\text {in }}}=0, \quad \text { for } \varphi=(2 n+1) \pi,
\end{gathered}
$$

where $n$ is any integer.

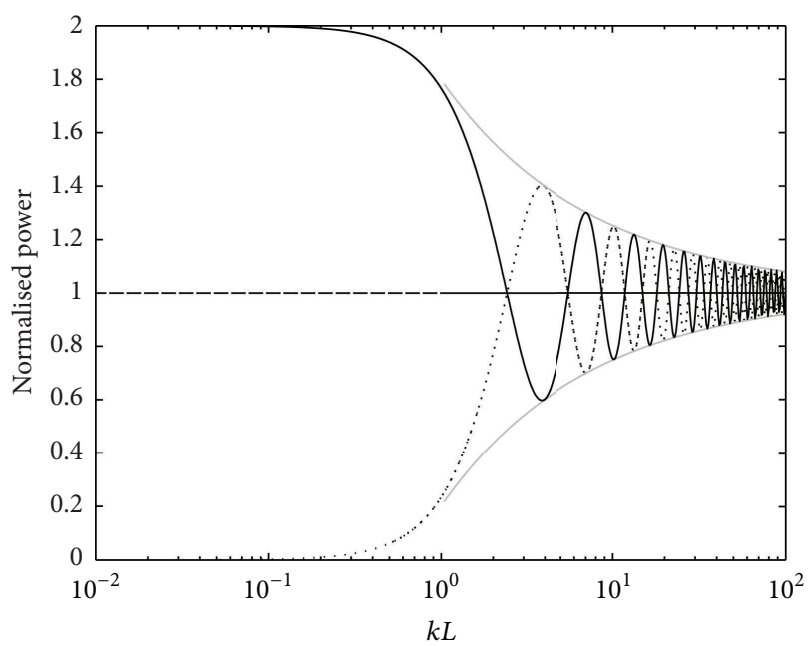

FIGURE 1: The normalised input power to an infinite plate subjected to two in-phase (-) and out-of-phase $(\cdots)$ harmonic unit point forces ( - grey line: $\mathrm{max} / \mathrm{min}$ bounds at higher frequencies).

When the structural wavelength is much smaller than $L$ $(\lambda \ll L)$, the input power is

$$
P_{\text {in }}=\left(\operatorname{Re}\left\{\widetilde{Y}_{p}\right\}+\operatorname{Re}\left\{\widetilde{Y}_{t}\right\} \cos \varphi\right) F^{2} .
$$

For small structural wavelength, the maximum and minimum input powers depend on $\operatorname{Re}\left\{Y_{t}\right\} \cos \varphi$ which indicates the dependency of the power on the phase and the distance between the excitation forces. Now since $k L \gg 1$, the real part of the transfer mobility in (6) can be expressed in its asymptotic form [16] as (see Appendix A)

$$
\operatorname{Re}\left\{\widetilde{Y}_{t}\right\}=\operatorname{Re}\left\{\widetilde{Y}_{p}\right\} \sqrt{\frac{2}{\pi k L}} \cos \left(k L-\frac{\pi}{4}\right) .
$$

The input power can thus be written as

$$
P_{\text {in }}=\operatorname{Re}\left\{\tilde{Y}_{p}\right\}\left(1+\sqrt{\frac{2}{\pi k L}} \cos \left(k L-\frac{\pi}{4}\right) \cos \varphi\right) F^{2} .
$$

The input power at higher frequencies is thus bounded by

$$
\overline{\overline{P_{\text {in }}}}=\left(1 \pm \sqrt{\frac{2}{\pi k L}}\right) \operatorname{Re}\left\{\tilde{Y}_{p}\right\} F^{2} .
$$

Figure 1 shows the total input power when the two forces are in-phase $(\varphi=0)$ and out-of-phase $(\varphi=\pi)$ as a function of $k L$. Note that the input power has been normalised with respect to the input power from two point forces acting incoherently (equal to two times the power from a single point force). It can be seen that the power fluctuates around the value it would have if the two forces were applied independently. From (12), the power is minimum and maximum when $k L=(2 n+1) \pi$ for $n=0,1, \ldots$ for in-phase and outof-phase forces, respectively. These are when, with respect to the wavelength, the two in-phase forces become out of phase and the out of phase forces become in phase. The intersection between the two curves at high $k L$ is when $\varphi=\pi / 2$. For $k L<$ 1 , that is, when the forcing distance is less than half a structural wavelength, the total power is constant with frequency. 
3.2. Random Phase. In practice, accurate information regarding the relative phase between the forces is generally not known. It might be assumed that all possible relative phases have equal probability. The probability density function $\Pi$ of the phase is then constant and given by

$$
\Pi(\varphi)=\frac{1}{2 \pi}, \quad \varphi=[0,2 \pi]
$$

From (10), the mean power and its variance using (5) are

$$
\begin{aligned}
& \mu_{P_{\text {in }}}=\operatorname{Re}\left\{\tilde{Y}_{p}\right\} F^{2}, \\
& \sigma_{P_{\text {in }}}^{2}=\frac{1}{2}\left(\operatorname{Re}\left\{\widetilde{Y}_{t}\right\} F^{2}\right)^{2} .
\end{aligned}
$$

It can be seen that the mean power equals that which would be given by two forces acting independently. The variance, hence, arises from interference between the two forces. As $\widetilde{Y}_{t}=\widetilde{Y}_{p}$ for $k L \ll 1$, from (15), the normalised standard deviation is $\sigma / \mu=1 / \sqrt{2}$. For $k L \gg 1, \sigma$ decreases as $k L$ increases (see (11)). Substituting (11) into (15) gives

$$
\frac{\sigma^{2}}{\mu^{2}}=\left(\frac{1}{\pi k L}\right) \cos ^{2}\left(k L-\frac{\pi}{4}\right) .
$$

The bounds of the variance occur when $\cos ^{2}(k L-\pi / 4)=1$. The maximum normalised standard deviation is therefore $\bar{\sigma} / \mu=1 / \sqrt{\pi k L}$.

When the separation distance of the contact points is uncertain, consequently $k L \bmod \pi$ becomes unknown, while $1 /(k L)$ is more or less constant. Therefore by averaging (16) over all possible $k L \bmod \pi$, the variance can be expressed as

$$
\begin{aligned}
\left\langle\frac{\sigma^{2}}{\mu^{2}}\right\rangle_{k L} & =\left(\frac{1}{\pi k L}\right)\left[\frac{1}{\pi} \int_{-\pi / 2}^{\pi / 2} \cos ^{2}\left(k L-\frac{\pi}{4}\right) \mathrm{d}(k L)\right] \\
& =\frac{1}{2 \pi k L}
\end{aligned}
$$

Therefore, the normalised standard deviation due to uncertainty in $k L$ is $\sigma / \mu=1 / \sqrt{2 \pi k L}$.

Figure 2 shows the input power for a number of possible relative phases of excitation. The mean power is a function of the point mobility which then gives a constant value with frequency. The normalised deviation of the power $(1 \pm \sigma / \mu)$ lies between the maximum and minimum input power and bounded by $1 \pm 1 / \sqrt{\pi k L}$. It can be seen that uncertainty in $k L$ increases the deviation of the input power.

3.3. Four Contact Points. The case of four input excitations is more realistic in practice, for example, a vibrating machine with four feet. Figure 3 shows an infinite plate excited by four harmonic point forces. In this case, from (1), the mobility is a $4 \times 4$ matrix. The diagonal elements are the point mobilities at each location, which for an infinite structure are equal to $\tilde{Y}_{p}$. The analysis is generally complicated. For simplicity, by assuming that the excitation positions form a rectangular

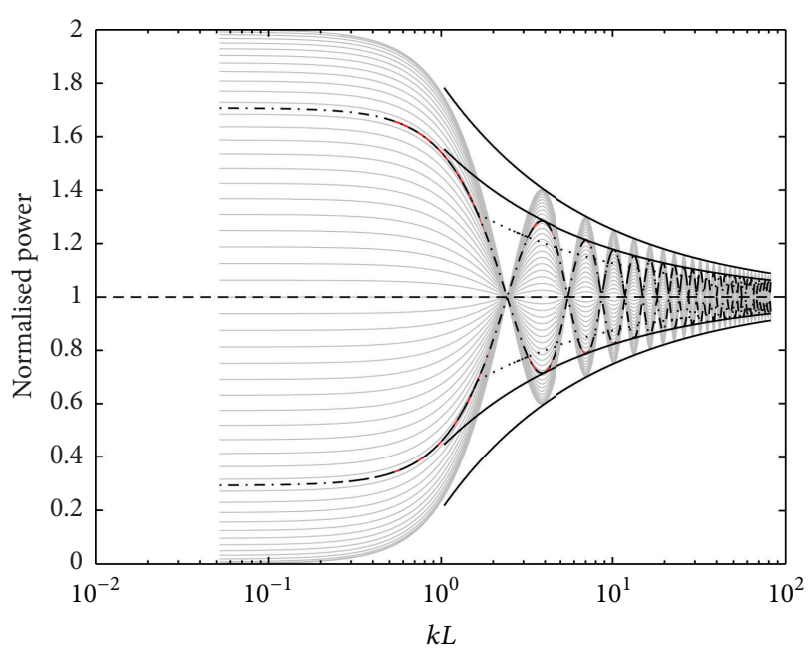

FIGURE 2: The normalised input power to an infinite plate subjected to two harmonic unit point forces with various relative phases (grey lines): -- mean, $-\cdot-$ mean \pm standard deviation, - (thick lines) $\mathrm{max} / \mathrm{min}$ bounds, - mean \pm bounds of standard deviation, and ... mean \pm bounds of standard deviation due to uncertainty in $k L$.

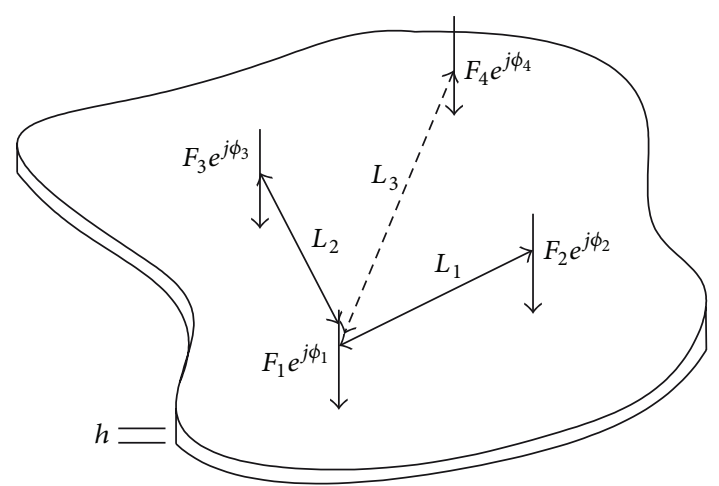

FIGURE 3: An infinite plate excited by four harmonic point forces applied at the vertices of a rectangle.

shape, therefore, $\tilde{Y}_{12}=\tilde{Y}_{21}=\tilde{Y}_{34}=\tilde{Y}_{43} ; \tilde{Y}_{13}=\tilde{Y}_{31}=\tilde{Y}_{24}=$ $\widetilde{Y}_{42}$ and $\widetilde{Y}_{14}=\tilde{Y}_{41}=\widetilde{Y}_{23}=\tilde{Y}_{32}$. The problem can be simplified numerically by choosing a force vector whose phases are relative to the phase of one reference force. The force vector can be rewritten as

$$
\widetilde{\mathbf{F}}=\left[\begin{array}{llll}
F_{1} & F_{2} e^{j \psi_{2}} & F_{3} e^{j \psi_{3}} & F_{4} e^{j \psi_{4}}
\end{array}\right]^{T},
$$

where $\psi_{i}$ is the relative phase of $F_{i}$ with respect to $F_{1}$. Thus, the total input power is given by

$$
\begin{aligned}
P_{\text {in }}= & \frac{1}{2} \operatorname{Re}\left\{\widetilde{Y}_{p}\right\}\left(F_{1}^{2}+F_{2}^{2}+F_{3}^{2}+F_{4}^{2}\right) \\
& +\operatorname{Re}\left\{\widetilde{Y}_{12}\right\}\left(F_{1} F_{2} \cos \psi_{2}+F_{3} F_{4} \cos \left(\psi_{3}-\psi_{4}\right)\right) \\
& +\operatorname{Re}\left\{\widetilde{Y}_{13}\right\}\left(F_{1} F_{3} \cos \psi_{3}+F_{2} F_{4} \cos \left(\psi_{2}-\psi_{4}\right)\right) \\
& +\operatorname{Re}\left\{\widetilde{Y}_{14}\right\}\left(F_{1} F_{4} \cos \psi_{4}+F_{2} F_{3} \cos \left(\psi_{2}-\psi_{3}\right)\right) .
\end{aligned}
$$


Note again in (19), that the input power is the sum of the powers that would be injected by the forces acting individually (the first term) and terms that depend on the relative phases of the forces (the remaining terms).

Thus, if equal probability of relative phase is assumed and the phases are uncorrelated, the probability density function $\Pi$ can be expressed as

$$
\begin{gathered}
\Pi\left(\psi_{2}, \psi_{3}, \psi_{4}\right)=\Pi\left(\psi_{2}\right) \Pi\left(\psi_{3}\right) \Pi\left(\psi_{4}\right), \\
\Pi\left(\psi_{i}\right)=\frac{1}{2 \pi} .
\end{gathered}
$$

Using (5) and assuming all the forces have equal amplitudes, the mean power and the variance are given by

$$
\begin{gathered}
\mu_{P_{\text {in }}}=2 \operatorname{Re}\left\{\widetilde{Y}_{p}\right\} F^{2}, \\
\sigma_{P_{\text {in }}}^{2}=\frac{1}{2}\left(\operatorname{Re}\left\{\widetilde{Y}_{12}\right\} F^{2}\right)^{2}+\frac{1}{2}\left(\operatorname{Re}\left\{\widetilde{Y}_{13}\right\} F^{2}\right)^{2} \\
+\frac{1}{2}\left(\operatorname{Re}\left\{\widetilde{Y}_{14}\right\} F^{2}\right)^{2} \\
+\frac{1}{2}\left(\operatorname{Re}\left\{\widetilde{Y}_{23}\right\} F^{2}\right)^{2}+\frac{1}{2}\left(\operatorname{Re}\left\{\widetilde{Y}_{24}\right\} F^{2}\right)^{2} \\
+\frac{1}{2}\left(\operatorname{Re}\left\{\tilde{Y}_{34}\right\} F^{2}\right)^{2} .
\end{gathered}
$$

As in the case of two point forces in Section 3.2, the variance of the input power depends only on the transfer mobilities while the mean power is only a function of the point mobility. In general, the mean and the variance of input power averaged over all possible phases of excitation for $N$ contact points for a rectangular separation can be written as

$$
\begin{gathered}
\mu_{P_{\text {in }}}=\frac{1}{2} \sum_{i}^{N} \operatorname{Re}\left\{\tilde{Y}_{i i}\right\} F_{i}^{2}, \\
\sigma_{P_{\text {in }}}^{2}=\frac{1}{2} \sum_{i=1}^{N-1} \sum_{\substack{k=2 \\
i<k}}^{N}\left(\operatorname{Re}\left\{\widetilde{Y}_{i k}\right\} F_{i} F_{k}\right)^{2} .
\end{gathered}
$$

The bounds for the normalised input power are obtained by inserting the maximum transfer mobility from (11) into (19), which for equal force amplitudes yields

$$
\frac{\overline{P_{\text {in }}}}{\bar{\mu}}=1 \pm \sqrt{\frac{2}{\pi k}}\left(L_{1}^{-1 / 2}+L_{2}^{-1 / 2}+L_{3}^{-1 / 2}\right) \text {. }
$$

In the same way, substituting (11) into (22), the bounds for the normalised standard deviation are

$$
\frac{\overline{\bar{\sigma}}}{\mu}= \pm \sqrt{\frac{1}{2 \pi k}}\left(L_{1}^{-1}+L_{2}^{-1}+L_{3}^{-1}\right)^{1 / 2},
$$

where the positive and negative signs are for the maximum and minimum bounds, respectively.

Following the same method for the case of two point forces, the bounds due to uncertainty in $k L$ are given by

$$
\frac{\bar{\sigma}}{\mu}= \pm 0.5 \sqrt{\frac{1}{\pi k}}\left(L_{1}^{-1}+L_{2}^{-1}+L_{3}^{-1}\right)^{1 / 2} .
$$

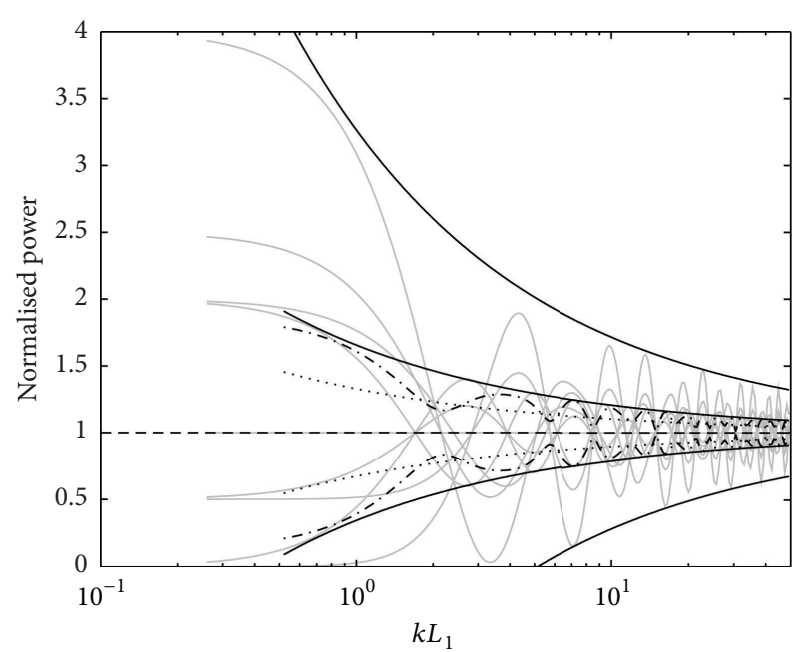

FIGURE 4: The normalised input power to an infinite plate subjected to four harmonic unit point forces with various phases (grey lines): -- mean, $-\cdot-$ mean \pm standard deviation, - (thick lines) $\mathrm{max} / \mathrm{min}$ bounds, - mean \pm bounds of standard deviation, and $\cdots$ mean \pm bounds of standard deviation due to uncertainty in $k L$.

Figure 4 shows the input power with various relative phases of excitation. As in Figure 2, the power fluctuates at high $k L$ around the mean value. The standard deviation shown is from (22). The first dip in the standard deviation corresponds to the frequency where the diagonal $L_{3}$ equals half the structural wavelength, and the subsequent dips are related to $L_{1}$ and $L_{2}$. Equations (25) and (26) again show that the standard deviation reduces as the separation of the contact points increases.

\section{Input Power to a Finite Plate}

For a finite structure, the mobility and, hence, the input power can be expressed in terms of a summation over modes of vibration. The mobility of a finite structure at an arbitrary point $(x, y)$ subjected to a point force $F$ at $\left(x_{0}, y_{0}\right)$ at frequency $\omega$ is given by [10]

$$
\tilde{Y}=j \omega \sum_{n=1}^{\infty} \frac{\Phi_{n}\left(x_{0}, y_{0}\right) \Phi_{n}(x, y)}{\omega_{n}^{2}\left(1+j \eta_{n}\right)-\omega^{2}},
$$

where $\Phi_{n}$ is the $n$th mass-normalised mode shape of the structure, $\omega_{n}$ is the $n$th natural frequency, and $\eta_{n}$ is damping loss factor of the $n$th mode. A case often considered is that of a rectangular plate with simply supported boundary conditions as this system provides a simple analytical solution. For a simply supported rectangular plate with dimensions $a \times b$, the mode shape and the natural frequency for mode $(p, q)$ are

$$
\begin{gathered}
\Phi_{p q}(x, y)=\frac{2}{\sqrt{M}} \sin \left(\frac{p \pi x}{a}\right) \sin \left(\frac{q \pi x}{b}\right), \\
\omega_{p q}=\sqrt{\frac{B}{m}}\left[\left(\frac{p \pi}{a}\right)^{2}+\left(\frac{q \pi}{b}\right)^{2}\right],
\end{gathered}
$$


where $M$ is the total mass of the plate and $p, q=1,2,3, \ldots$. The rigid body motion existing in the reception plate method [11] can be approximated by assuming the edges are guided or sliding. The mode shape functions involve cos terms instead of sin terms in (28). Using the corresponding real part of the point mobility in (27), the input power for point excitation is given by

$$
P_{\text {in }}=\frac{1}{2} \sum_{p=1}^{\infty} \sum_{q=1}^{\infty} \frac{\omega \omega_{p q}^{2} \eta_{n} \Phi_{p q}^{2}\left(x_{0}, y_{0}\right)|F|^{2}}{\left(\omega_{p q}^{2}-\omega^{2}\right)^{2}+\left(\omega_{p q}^{2} \eta_{n}\right)^{2}}
$$

4.1. Averaging over Force Positions. Equation (29) implies that the input power depends on the forcing location which might be uncertain. Figure 5 shows the input power from a single point force for various forcing locations. The structure considered is an aluminium plate having dimensions $0.6 \times$ $0.5 \times 0.0015 \mathrm{~m}$, Young's modulus $7.1 \times 10^{10} \mathrm{~N} / \mathrm{m}^{2}, \rho=$ $2700 \mathrm{~kg} / \mathrm{m}^{3}$, and damping loss factor $\eta=0.05$, assumed constant for every mode. The input power is normalised with respect to the input power to an infinite plate.

The peaks occur at the resonances of the plate. They are distinct at low frequencies, but the modal overlap increases as the frequency increases. Analytically, since $1 /(a b) \int_{a} \int_{b} \Phi_{p q}^{2} \mathrm{~d} x_{0} \mathrm{~d} y_{0}=1 / M$, the mean input power averaged over all possible force positions is [10] as follows:

$$
\left\langle P_{\text {in }}\right\rangle_{x_{0}, y_{0}}=\frac{|F|^{2}}{2 M} \sum_{p=1}^{\infty} \sum_{q=1}^{\infty} \frac{\omega \omega_{p q}^{2} \eta}{\left(\omega_{p q}^{2}-\omega^{2}\right)^{2}+\left(\omega_{p q}^{2} \eta\right)^{2}} .
$$

It can be seen that at high frequencies, the mean power converges to the same level as the input power to an infinite plate. The variance of the input power also decreases at high frequencies as with respect to the spatial variation of the vibration modes, the forcing location becomes less important as the frequency increases.

4.2. Averaging over Frequency Bands. Suppose that the excitation frequency lies between two frequencies $\omega_{1}$ and $\omega_{2}$. The input power can then be averaged over this frequency band and can be expressed as

$$
\left\langle P_{\text {in }}\right\rangle_{\omega}=\frac{1}{\omega_{2}-\omega_{1}} \int_{\omega_{2}}^{\omega_{1}} P_{\text {in }}(\omega) \mathrm{d} \omega .
$$

Figure 6 shows the average input power for a $100 \mathrm{~Hz}$ bandwidth and at $50 \mathrm{~Hz}$ centre frequency spacing. For the plate example considered, the modal density is 0.065 modes/Hz. Therefore with this bandwidth, there are on average just over 6 modes in the band. It can be seen that the mean value is now close to the infinite plate value except below $100 \mathrm{~Hz}$, where the modal overlap is low and the response is stiffness dominated.

4.3. Prediction of Mean and Variance. The frequency average of the input power in (29) strongly depends on the statistical distribution of $\omega /\left(\left(\omega_{m n}^{2}-\omega^{2}\right)^{2}+\left(\omega_{m n}^{2} \eta\right)^{2}\right)$. For a symmetric structure like a rectangular plate, asymptotically the natural frequency spacings have a Poisson distribution; that is, the

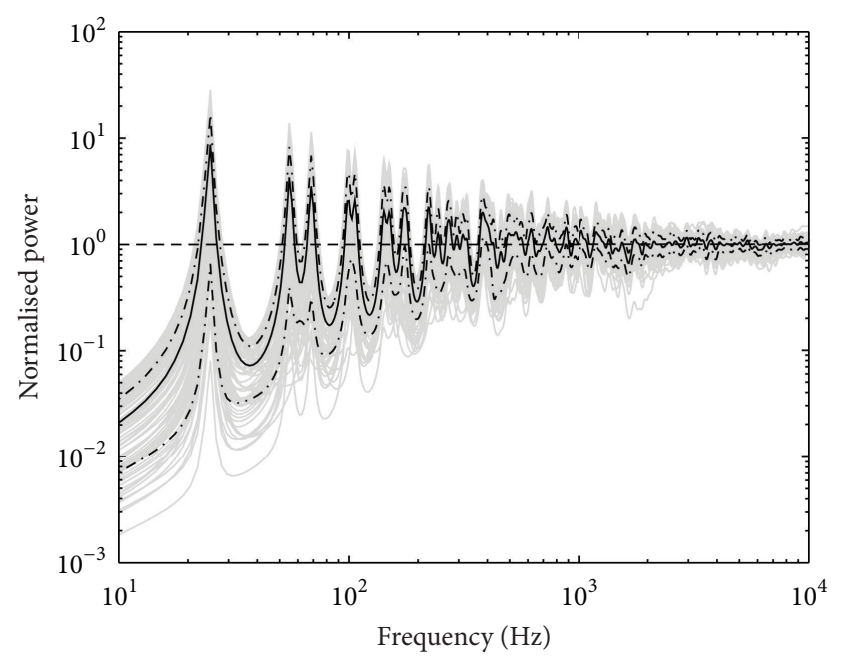

FIGURE 5: The normalised input power to a finite plate subjected to a single harmonic point force for various possible force positions (grey lines): - mean and $-\cdot-$ mean \pm standard deviation.

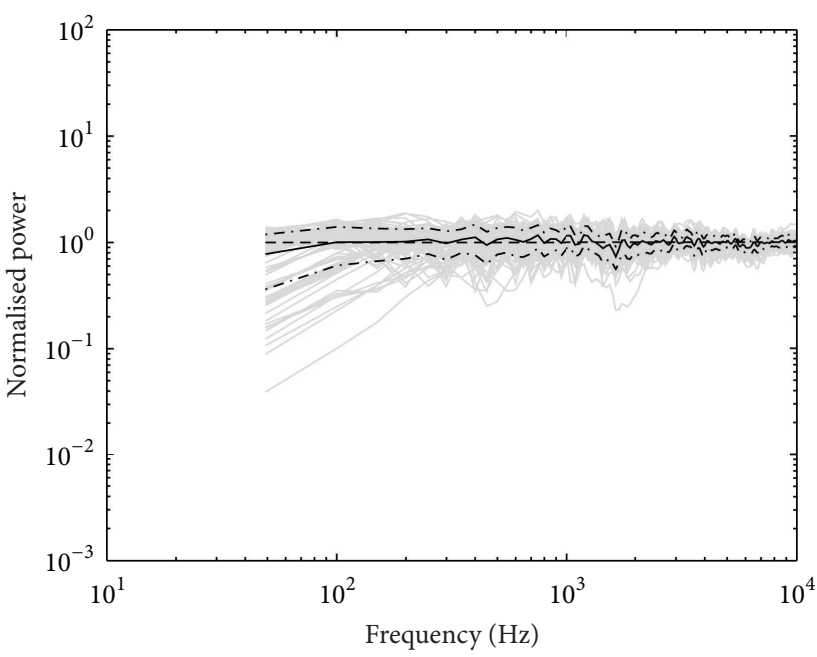

FIGURE 6: The normalised input power to a finite plate subjected to a single harmonic point force for various possible force positions averaged over $100 \mathrm{~Hz}$ frequency band (grey lines): - mean and - - mean \pm standard deviation.

spacings between successive natural frequencies are statistically independent and have an exponential distribution [17]. From $[1,2,10,17]$, the mean and variance of the input power averaged over all possible forcing locations yield

$$
\begin{aligned}
& \left\langle P_{\text {in }}\right\rangle_{\left(\omega, x_{0}, y_{0}\right)}=\frac{|F|^{2} n_{d} \pi}{4 M}, \\
& \left\langle\sigma^{2}\right\rangle_{\left(\omega, x_{0}, y_{0}\right)}=\frac{|F|^{4} n_{d} \pi}{16 \eta \omega M^{2}},
\end{aligned}
$$

where $n_{d}(\omega)=0.276 a b / h c_{L}$ is the modal density of plate and $c_{L}=\left(E / \rho\left(1-v^{2}\right)\right)^{1 / 2}$ is the longitudinal plate wave speed. The mean power equals the input power to an infinite plate. 

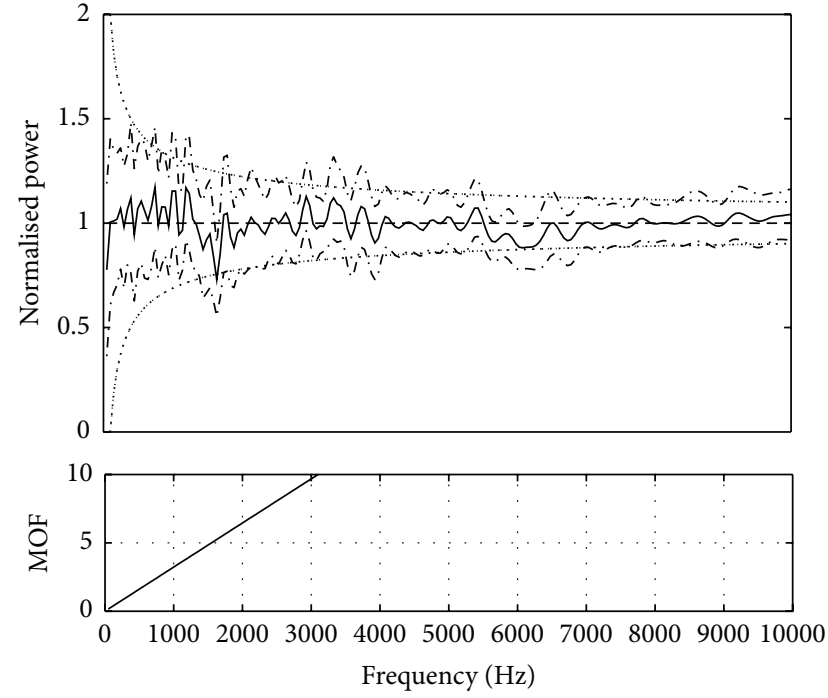

(a)
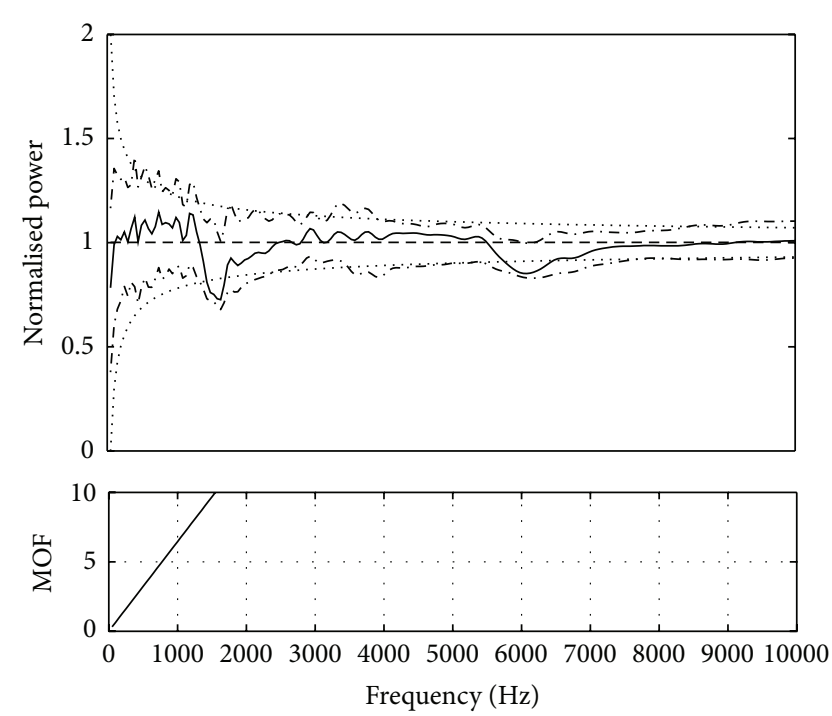

(b)

FIGURE 7: The mean and standard deviation of the input power of a finite plate due to variation in forcing locations (numerical: - - mean, - · mean \pm standard deviation, and (32): -- mean and $\cdots$ mean \pm standard deviation; (a) $\eta=0.05$ and (b) $\eta=0.1$ ).

Figure 7 shows the normalised standard deviation of the input power averaged over force positions for different damping loss factors together with the modal overlap factor (MOF), where MOF $=n_{d} \eta \omega$. It can be seen that there is a good agreement between the numerical calculations and the analytical predictions if MOF $>1$.

From (32), the relative standard deviation can be defined as a function of modal overlap factor; that is,

$$
r_{\sigma}=\frac{\langle\sigma\rangle}{\left\langle P_{\text {in }}\right\rangle}=\frac{1}{\sqrt{\pi n_{d} \eta \omega}}=\frac{1}{\sqrt{\pi \mathrm{MOF}}} .
$$

For plates with other shapes, the natural frequency spacing statistics are not Poisson (e.g., under many circumstances they asymptote to Gaussian orthogonal ensemble statistics). An alternative expression for the variance can then be found $[1,2]$.

4.4. Four Point Excitations. In this section, results are presented for the case where there are four rectangularly distributed point excitations. The diagram of the force positions is the same as in Figure 3 for an infinite plate. The total input power is also similar to (19) except that for a finite structure the leading term is now expressed in terms of the input mobility for each force location. Therefore by also assuming equal force amplitudes and equal probability of all the excitation phases, the mean power for a finite plate subjected to four point forces according to (23) is given by

$$
\begin{gathered}
\mu_{P_{\text {in }}}=\frac{1}{2}\left(\operatorname{Re}\left\{\widetilde{Y}_{11}\right\}+\operatorname{Re}\left\{\widetilde{Y}_{22}\right\}+\operatorname{Re}\left\{\widetilde{Y}_{33}\right\}\right. \\
\left.+\operatorname{Re}\left\{\widetilde{Y}_{44}\right\}\right) F^{2},
\end{gathered}
$$

whilst the variance is the same as in (22).

Figure 8 shows the normalised mean and the standard deviation of the input power to a plate having dimensions

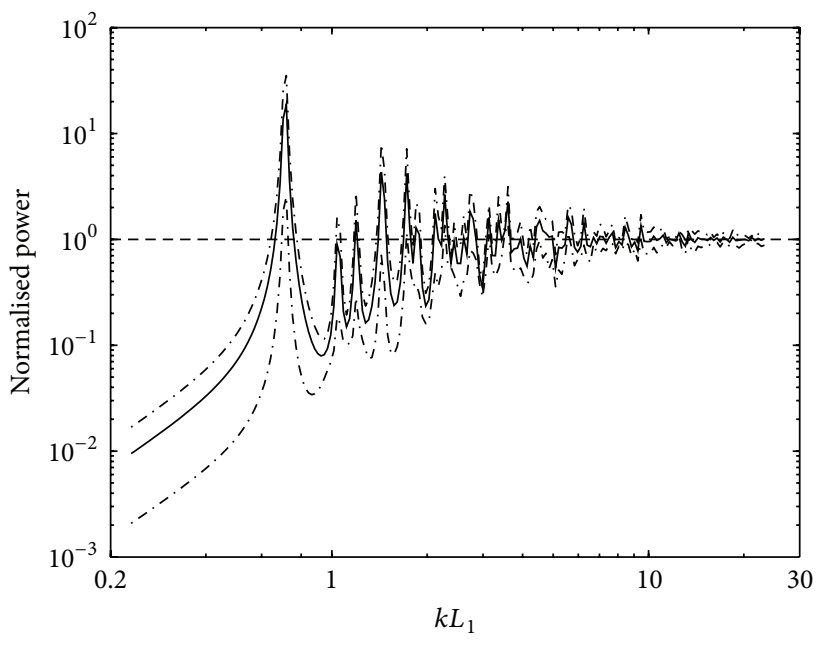

FIGURE 8: The normalised input power to a finite plate subjected to four harmonic point forces averaged over all possible excitation phases $(-$ mean and $-\cdot-$ mean \pm standard deviation).

$1 \times 0.8 \times 0.0015 \mathrm{~m}$. The simulation is made for forces with a square distribution, where $L_{1}=L_{2}=0.1 \mathrm{~m}$ (see Figure 3 ), and is located around the middle of the plate. The result shows typical behaviour where the variation is larger at low frequencies and decreases as $k L$ increases.

For the same force separation, Figure 9 shows the relative standard deviation $r_{\sigma}$ for the average input power over all possible force positions and frequency bands. It can be seen that the standard deviation can be estimated reasonably and accurately by using results for the infinite plate, except at very low $k L$. The result from (33) is close to the averages of the numerical results. The figure also shows that the dips clearly correspond to frequencies where the distance between 


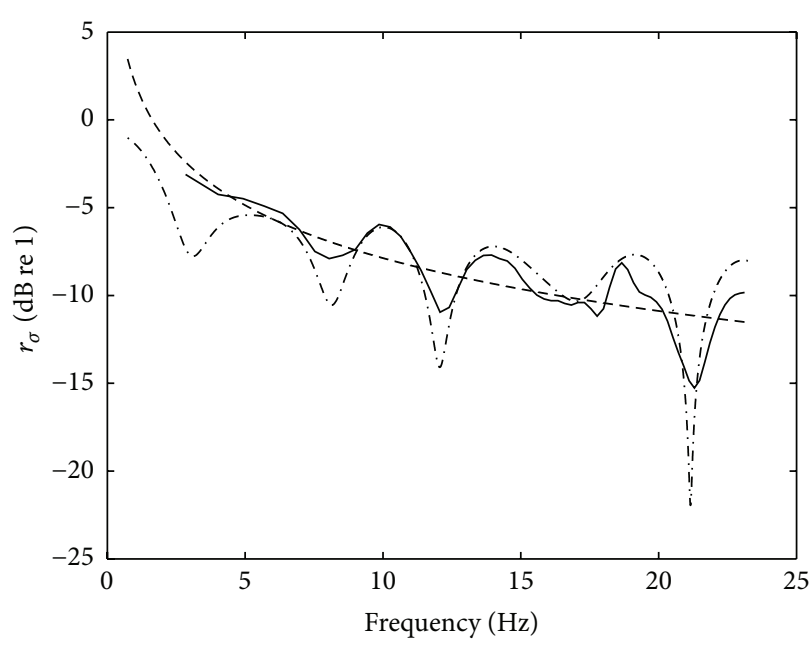

(a)

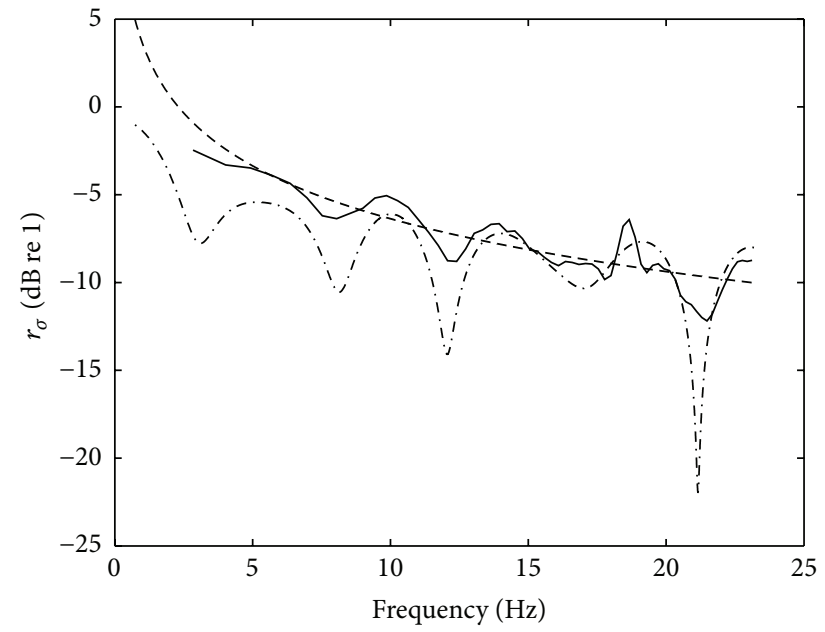

(b)

FIGURE 9: The relative standard deviation of input power to a finite plate subjected to four harmonic point forces averaged over various force positions and frequency bands: - numerical, $-\cdot-$ infinite plate and $--(33)((a) \eta=0.1$ and (b) $\eta=0.05)$.

the excitation points is a multiple of half the structural wavelength, $k L=n \pi$. However, Figure 9 (b) shows that for a smaller damping, the infinite plate result is now an underestimate of the numerical values. In this case, the prediction using (33) gives a good agreement, particularly at large $k L$.

\section{Random Force Amplitude}

Besides the phases and locations of excitation, the force distribution at the contact points also generates variability in the input power. In practice, this could be due to the condition of the installation or the nature of the source itself. The problem is that it is very difficult in practice to have knowledge of its distribution in sufficient detail. The situation becomes more complicated still if moment excitation is also taken into consideration. Thus, in theoretical studies often, for convenience, only translational forces of equal amplitude are assumed $[8,11]$.

Here, the approach is used to constrain the total square magnitude of the forces, that is, to assume

$$
F_{\mathrm{eff}}^{2}=\sum_{i=1}^{N} F_{i}^{2}
$$

is constant. The goal is to obtain the maximum and minimum bounds of the mean and variance of the input power providing that only the so-called effective force $F_{\text {eff }}$ is known. From (23) for the mean input power to an infinite plate excited by two point forces,

$$
\mu_{P_{\mathrm{in}}}=\frac{1}{2} \widetilde{Y}_{p}\left(F_{1}^{2}+F_{2}^{2}\right)=\frac{1}{2} \widetilde{Y}_{p} F_{\text {eff }}^{2}
$$

It can be seen that for an infinite plate, the mean power does not depend on the force distribution but rather on the characteristics of the receiver only. However, the variance depends on the product of the forces at the contact points. For two forces, the maximum variance is obtained when both contact points have equal force distribution; that is, $F_{1}=F_{2}$, and the minimum variance is when one of the forces equals the effective force while the other is zero; that is, $F_{1}=0 ; F_{2}=F_{\text {eff }}$ (see (23)). For a finite plate, the location of the excitation determines the mobility of the receiver. However, if the spatial variation is small $(k L<\pi)$, the mean input power also depends only on the receiver mobility as in (36), where $\widetilde{Y}_{p}=$ $\widetilde{Y}_{11} \approx \widetilde{Y}_{22}$.

Figure 10(a) shows the distribution of mean and the standard deviation of the input power due to random relative phases for various force amplitudes in frequency averages, where $F_{\text {eff }}^{2}=1 \mathrm{~N}^{2}$. The excitation points are located at $(0.25 a$, $0.6 b)$ and $(0.58 a, 0.6 b)$ for the same plate dimensions as in Section 4.1 with damping loss factor $\eta=0.01$. It can be seen that at low frequency, the variation is up to $4 \mathrm{~dB}$ which is quite significant. However, this reduces as the frequency increases. The variation can also be reduced by increasing the damping loss factors as shown in Figure 10(c).

The average mean and variance from this results can be obtained by assuming $F_{1}^{2}$ is uniformly probable between 0 and $F_{\text {eff. }}^{2}$. Within this range, the probability density function $\Pi$ can be assumed constant; that is,

$$
\begin{aligned}
& \Pi\left(F_{1}^{2}\right)=\frac{1}{F_{\text {eff }}^{2}}, \\
& F_{1}^{2}=\left[0, F_{\text {eff }}^{2}\right] .
\end{aligned}
$$

Using (5), the mean and the variance of the input power due to random phases averaged over random point force loading for the case of two contact points are given by

$$
\begin{aligned}
& \left\langle\mu_{P_{\text {in }}}\right\rangle_{F_{1}^{2}}=\frac{1}{4}\left(\operatorname{Re}\left\{\tilde{Y}_{11}\right\}+\operatorname{Re}\left\{\tilde{Y}_{22}\right\}\right) F_{\text {eff }}^{2}, \\
& \left\langle\sigma_{P_{\text {in }}^{2}}^{2}\right\rangle_{F_{1}^{2}}=\frac{1}{12}\left(\operatorname{Re}\left\{\widetilde{Y}_{12}\right\} F_{\text {eff }}^{2}\right)^{2} .
\end{aligned}
$$




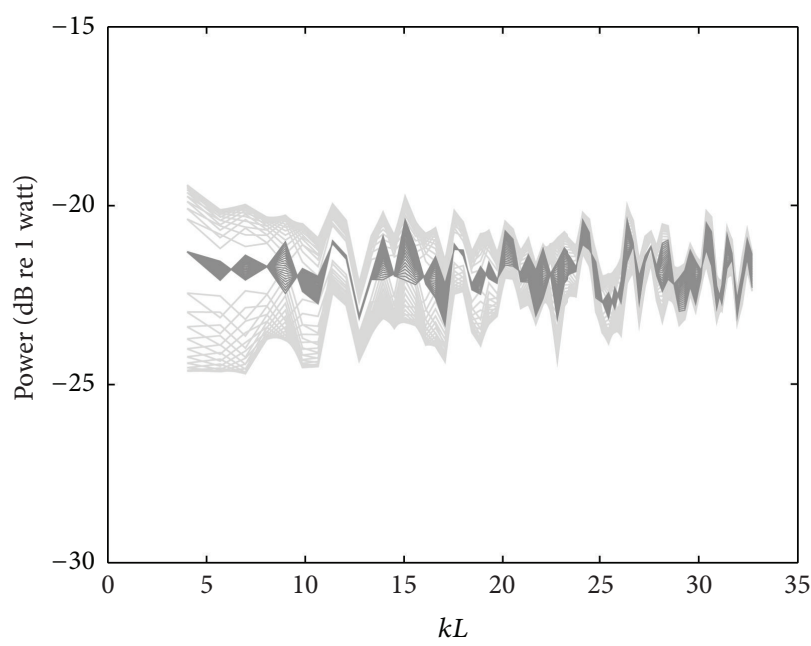

(a)

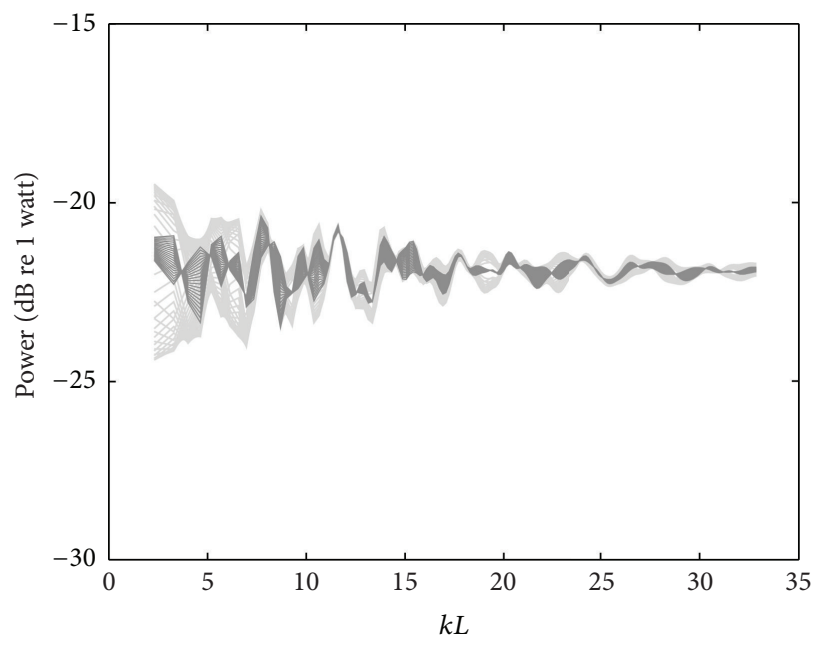

(c)

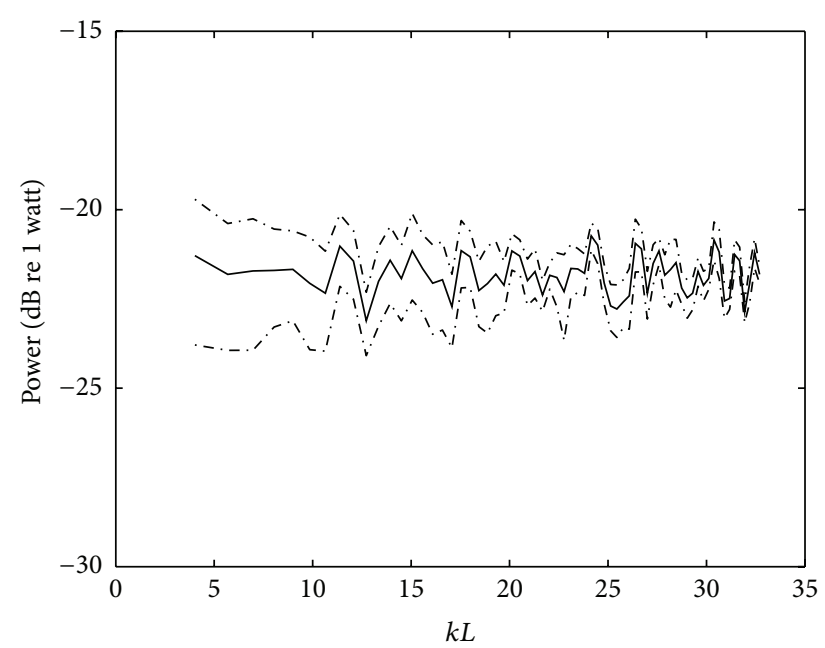

(b)

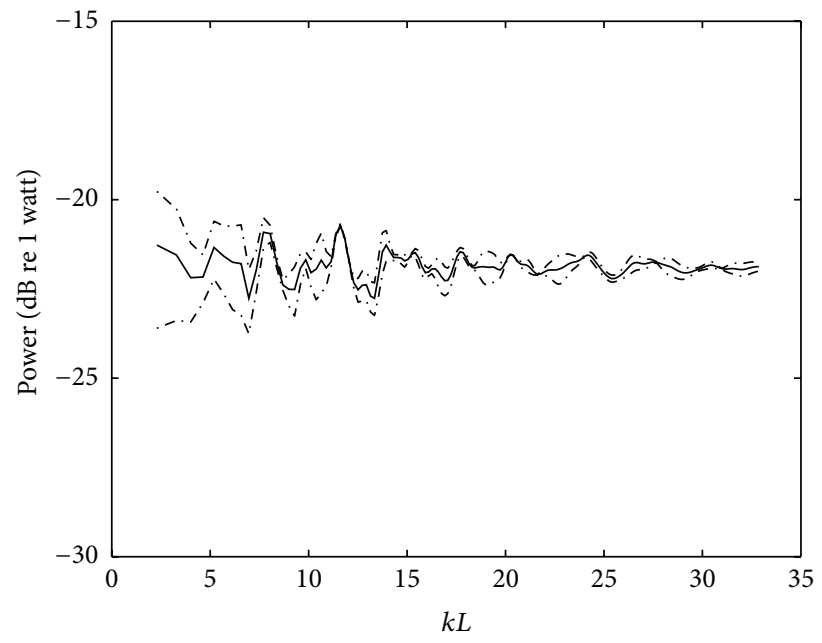

(d)

Figure 10: (a, c) The distribution of the mean (dark grey) and mean \pm standard deviation (light grey) of the input power to a finite plate with two contact points due to random relative phases for various force amplitudes; (b, d) The average mean ( - ) and average mean \pm standard deviation $(-\cdot-)$ over random force amplitudes. (a)-(b) $\eta=0.01$; (c)-(d) $\eta=0.1$.

Figures $10(\mathrm{~b})$ and $10(\mathrm{~d})$ show the average mean and standard deviation of the input power from the results in Figures 10(a) and 10(c). Here, again it can be clearly seen that the deviation decreases with frequency and also as the damping loss factor increases. In this example, for $\eta=0.1$, the deviation of the power from the mean is insignificant above $k L=5(<1 \mathrm{~dB})$.

Figure 11 shows the mean and variance due to random phases and force amplitudes for the infinite plate, also with two contact points, where in (38), $\widetilde{Y}_{11}=\widetilde{Y}_{22}=\widetilde{Y}_{p}$. Asymptotic form of $\widetilde{Y}_{t}$ in (11) is used to calculate the maximum and minimum bounds (the case where $F_{1}^{2}=F_{2}^{2}=F_{\text {eff }}^{2} / 2$ ). The range between the bounds can be seen to be roughly $2 \mathrm{~dB}$ between $k L=4$ and 16. However, this decreases as the frequency increases.

\section{The Contribution of Moment Excitation}

6.1. The Effect of Moment Excitation on the Input Power. In the previous sections, only the translational force is considered as the driving excitation. However, in principle the motion at a contact point of a structure-borne source would involve up to six components where not only forces, but also moments will contribute to the total input power. The moment excitation is often neglected partly because of measurement difficulties rather than the fact that, in most cases, it gives a small contribution to the input power [18]. The contribution of moments is most important at higher frequencies and is less important than that of forces if the source is far away from discontinuities or boundaries [19].

Figure 12 illustrates the components of excitation assumed to act on a structure. The response at the contact 


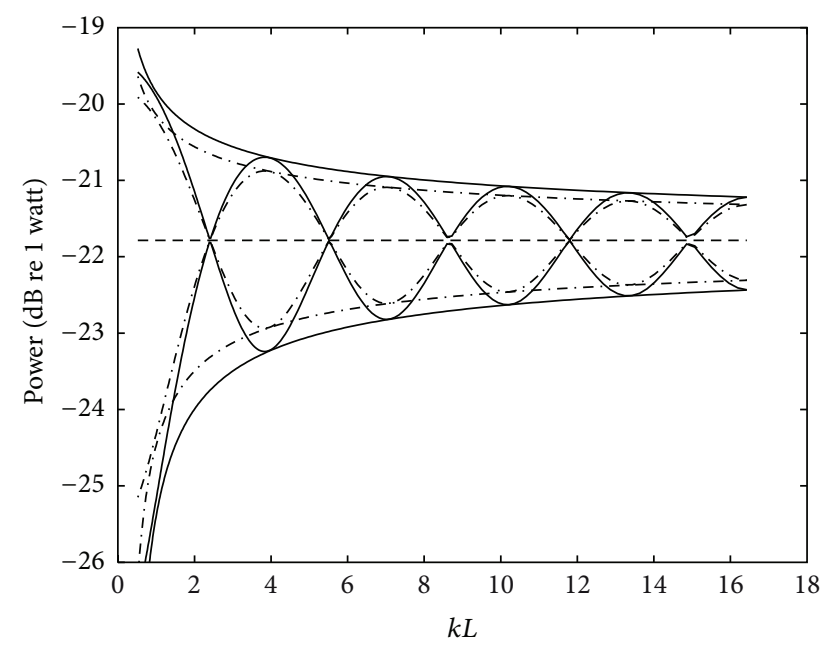

FIGURE 11: The mean $(--)$ and mean \pm standard deviation $(-\cdot-)$ of the input power to an infinite plate with two contact points due to random relative phases and random force amplitudes ( - max-min mean \pm standard deviation, (thick line) max-min bounds).

point is a function of point mobilities, transfer mobilities for different axes, and also the cross-mobilities for different components. Therefore, there will be a $6 \times 6$ mobility matrix for each excitation point. The problem becomes more complicated for multiple contact points. For $N$ contact points, the interaction between components will increase the size of the system matrix to $6 N \times 6 N$.

In this paper, however, the problem is simplified by neglecting the in-plane excitations, that is, $F_{x}, F_{y}$, and $M_{z}$. Therefore, the mobility matrix is reduced to a $3 \times 3$ matrix for a single contact point. In general, the input power due to a combined point force and moment excitation can be rewritten as

$$
\begin{aligned}
P_{\text {in }}=\frac{1}{2}( & \operatorname{Re}\left\{\widetilde{Y}^{v F}\right\}|\widetilde{F}|^{2}+\operatorname{Re}\left\{\widetilde{Y}^{v M} \widetilde{M} \widetilde{F}^{*}\right\} \\
& \left.+\operatorname{Re}\left\{\widetilde{Y}^{\dot{\theta} F} \widetilde{F} \widetilde{M}^{*}\right\}+\operatorname{Re}\left\{\widetilde{Y}^{\dot{\theta} M}\right\}|\widetilde{M}|^{2}\right),
\end{aligned}
$$

where $\widetilde{Y}^{v F}$ and $\widetilde{Y}^{\dot{\theta} M}$ are the point force and point moment mobilities and $\widetilde{Y}^{\dot{\theta} F}$ and $\widetilde{Y}^{v M}$ are the cross-mobilities from force to rotation and from moment to translation, respectively. Since $\widetilde{Y}^{\dot{\theta} F}=\widetilde{Y}^{v M}$, hence $\operatorname{Re}\left\{\widetilde{Y}^{v M} \widetilde{M} \widetilde{F}^{*}\right\}+\operatorname{Re}\left\{\widetilde{Y}{ }^{\dot{\theta} F} \widetilde{F} \widetilde{M}^{*}\right\}=$ $\operatorname{Re}\left\{\widetilde{Y}^{v M}\left(\widetilde{M} \widetilde{F}^{*}+\widetilde{F} \widetilde{M}^{*}\right)\right\}=2 \operatorname{Re}\left\{\widetilde{Y}^{v M}\right\} \operatorname{Re}\left\{\widetilde{M} \widetilde{F}^{*}\right\}$, and (39) can be rewritten as

$$
\begin{aligned}
& P_{\text {in }}=\frac{1}{2}( \operatorname{Re}\left\{\widetilde{Y}^{v F}\right\}|\widetilde{F}|^{2}+2 \operatorname{Re}\left\{\widetilde{Y}^{v M}\right\} \operatorname{Re}\left\{\widetilde{M} \widetilde{F}^{*}\right\} \\
&\left.+\operatorname{Re}\left\{\widetilde{Y}^{\dot{\theta} M}\right\}|\widetilde{M}|^{2}\right)
\end{aligned}
$$

where, for an infinite plate, $\operatorname{Re}\left\{\widetilde{Y}^{v M}\right\}=0$.

In matrix form, the power can be expressed as in (1) where $\widetilde{\mathbf{F}}=\left[\begin{array}{lll}\widetilde{F} & \widetilde{M}_{x} & \widetilde{M}_{y}\end{array}\right]^{T}$ is the vector of the force and moments.

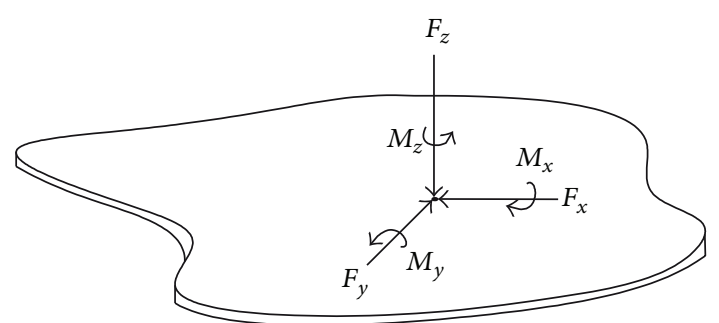

Figure 12: Six components of point excitations.

With inclusion of the moment excitation, the mobility matrix for a single contact point is given by

$$
\widetilde{\mathbf{Y}}=\left[\begin{array}{ccc}
\tilde{Y}^{v F} & \tilde{Y}^{v M_{x}} & \tilde{Y}^{v M_{y}} \\
\tilde{Y}^{\dot{x}_{x} F} & \tilde{Y}^{\dot{\theta}_{x} M_{x}} & \tilde{Y}_{x}^{\dot{\theta}_{x} M_{y}} \\
\tilde{Y}^{\dot{\theta}_{y} F} & \tilde{Y}^{\dot{\theta}_{y} M_{x}} & \tilde{Y}^{\dot{\theta}_{y} M_{y}}
\end{array}\right],
$$

where $\widetilde{\mathbf{Y}}$ is symmetric.

6.2. Magnitude of Moment. The relative contribution to the input power depends of course on the magnitude of the excitation. Force and moment cannot be compared directly as they have different units. In a practical situation, they would also depend on the nature of the force generation mechanism in the source. The installation condition also has to be considered. The effects of moment excitation for a vibrating machine installed on soft support at the contact points would be different to those if the machine was bolted tightly to the receiver structure. Thus, the problem remains of qualifying the relative effects of force and moment. Moorhouse [18] proposed a dimensionless mobility where, for example, the real part of a cross-mobility, $\operatorname{Re}\left\{\widetilde{Y}^{\dot{\theta} F}\right\}$, is normalised by the real part of the corresponding point mobilities for both the force and the moment, $\sqrt{\operatorname{Re}\left\{\widetilde{Y}^{\dot{\theta} M}\right\} \operatorname{Re}\left\{\widetilde{Y}^{v F}\right\}}$. This gives insight into the relative contribution due to the different excitation components.

6.2.1. Single Point Excitation. The relative importance of force and moment in exciting a structure can be compared only in terms of their input power. However, to calculate the power not only the mobilities should be known but also the magnitudes and the phases of the excitation components (see (39)). Petersson [20] introduced the nondimensional eccentricity which relates the ratio of magnitude of moment and force to the structural wavenumber.

Here, another approach is introduced where the magnitudes of the moments, $\widetilde{M}=M e^{j \phi_{1}}$, and the force, $\widetilde{F}=F e^{j \phi_{2}}$, at the contact point are related by an effective lever $\operatorname{arm} \alpha$ by

$$
M=\alpha F,
$$

where $0<\alpha<\infty$. This indicates that if $\alpha$ is very small, the structure is excited mainly by force, while if $\alpha$ is very large the structure is driven mainly by a moment. However, for convenience, a nondimensional unit is preferred to scale the 
relative input power. The total input powers, $P_{F}$ and $P_{M}$, due to a force and a moment on an infinite plate are

$$
P_{\text {in }}=P_{F}+P_{M}=\frac{1}{2} \operatorname{Re}\left\{\widetilde{Y}^{v F}\right\} F^{2}+\frac{1}{2} \operatorname{Re}\left\{\tilde{Y}^{\dot{\theta} M}\right\} M^{2},
$$

and the real parts of the point mobilities are given by

$$
\begin{aligned}
& \operatorname{Re}\left\{\tilde{Y}^{\nu F}\right\}=\frac{\omega}{8 B k^{2}}, \\
& \operatorname{Re}\left\{\tilde{Y}^{\dot{\theta} M}\right\}=\frac{\omega}{8 B},
\end{aligned}
$$

where all the cross-mobilities $\operatorname{Re}\left\{\widetilde{Y}^{\dot{\theta} F}\right\}$ and $\operatorname{Re}\left\{\widetilde{Y}^{v M}\right\}$ are zero. Consequently, the relative phase between the force and the moment is irrelevant.

From (42), (43), and (44), the input power from moment excitation can be scaled in terms of the input power from the force by a nondimensional unit $k \alpha$ and is expressed as

$$
P_{M}=(k \alpha)^{2} P_{F},
$$

where $k$ is the structural wavenumber. Equation (43) can be rewritten as

$$
P_{\text {in }}=\left((k \alpha)^{2}+1\right) P_{F} .
$$

Figure 13 shows the normalised total input power to an infinite plate for a single contact point. It can be seen that the power from force excitation is constant with frequency while the power from moment excitation is increasing with frequency. Both powers intersect at $k \alpha=1$. For $k \alpha<1$, the power is dominated by force excitation and for $k \alpha>1$, the power is dominated by moment excitation.

For a finite plate receiver, the total input power is given as in (40). While the situation is now numerically complicated, (46) can again be used to scale the individual contribution to the input power.

6.2.2. Multiple Point Excitation. Figure 14 shows a diagram of a translational force $F$ which generates moment $M$ that can be resolved into moments $M_{x}$ and $M_{y}$ components. The moments can be expressed as

$$
\begin{aligned}
& M_{x}=L \beta F \sin (\delta), \\
& M_{y}=-L \beta F \cos (\delta),
\end{aligned}
$$

where $L$ is the lever arm, or the distance from the line of action of $F$ to the point attached to the structure, $\delta$ is the angle between the lever arm and the positive $x$-axis, and $\beta$ is a dimensionless scaling factor.

Equations (42) and (47) can be used to define the relation between force and moment for multiple contact points. Figure 15 shows the forces and moments for a typical four point contact source, with the points having a rectangular distribution, where $L_{3}^{2}=L_{1}^{2}+L_{2}^{2}$. The reference moment at any contact point might then be considered as a sum of contributions from forces at all the contact points. In this

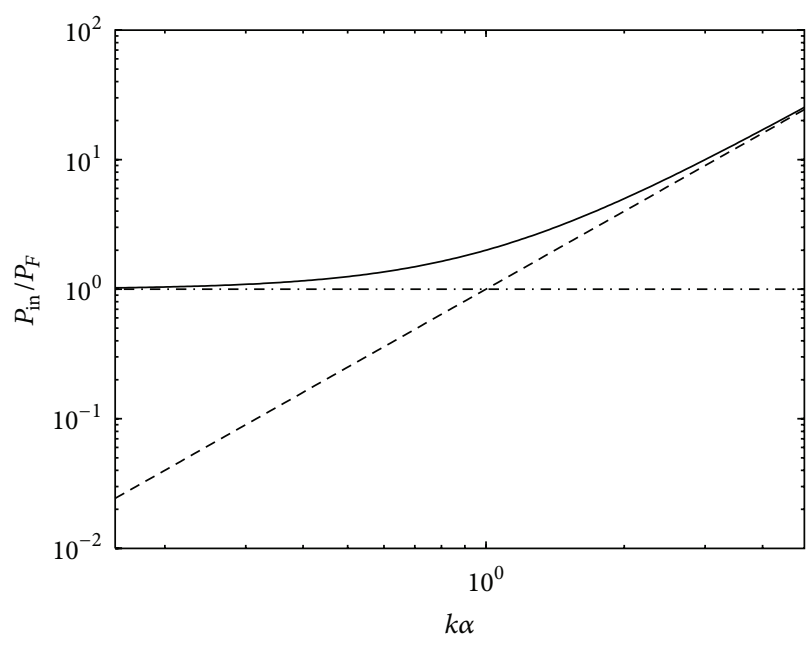

Figure 13: The normalised input power from force $(-\cdot-)$ and moment (--) excitations at a single contact point and the total power $(-)$.

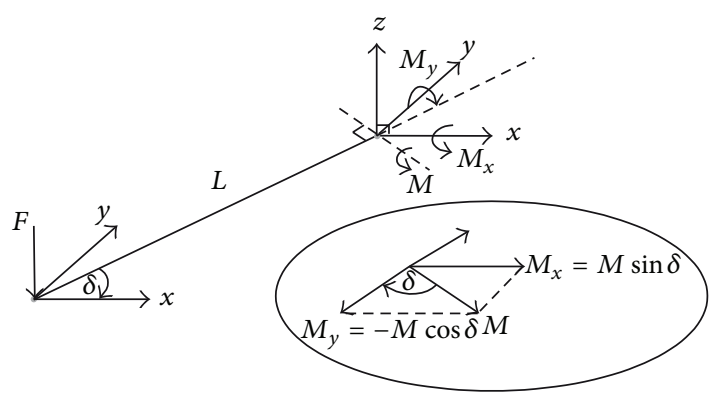

FIGURE 14: The lever arm of the moment and force.

situation, the moment about the $x$-axis can thus be expressed in the form

$$
\left\{\begin{array}{l}
M_{x, 1} \\
M_{x, 2} \\
M_{x, 3} \\
M_{x, 4}
\end{array}\right\}
$$

$$
\begin{aligned}
= & {\left[\begin{array}{cccc}
\alpha_{1} & 0 & -L_{2} \beta_{3} & -L_{3} \beta_{4} \sin \theta \\
0 & \alpha_{2} & -L_{3} \beta_{3} \sin \theta & -L_{2} \beta_{4} \\
L_{2} \beta_{1} & L_{3} \beta_{2} \sin \theta & \alpha_{3} & 0 \\
L_{3} \beta_{1} \sin \theta & L_{2} \beta_{2} & 0 & \alpha_{4}
\end{array}\right] } \\
& \times\left\{\begin{array}{l}
F_{1} \\
F_{2} \\
F_{3} \\
F_{4}
\end{array}\right\},
\end{aligned}
$$




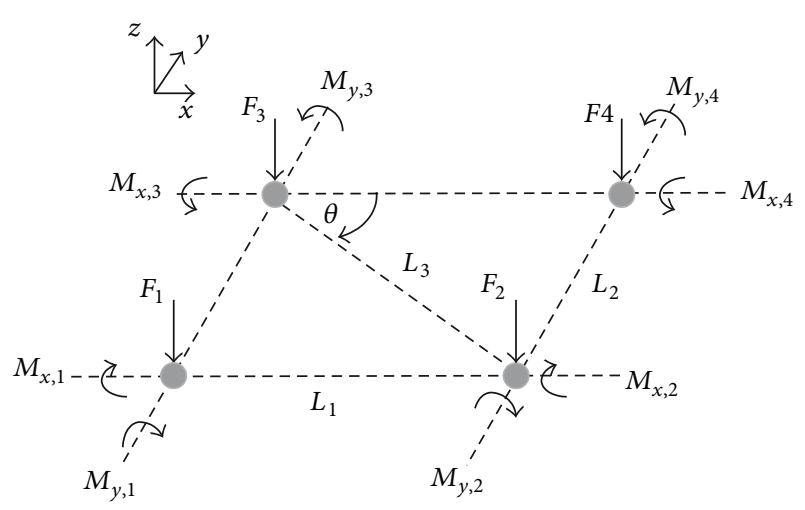

FIGURE 15: The moment and force directions at source-receiver interface with four contact points.

and the moments about the $y$-axis are

$$
\begin{aligned}
& \left\{\begin{array}{l}
M_{y, 1} \\
M_{y, 2} \\
M_{y, 3} \\
M_{y, 4}
\end{array}\right\} \\
& =\left[\begin{array}{cccc}
\alpha_{1} & L_{1} \beta_{2} & 0 & L_{3} \beta_{4} \cos \theta \\
-L_{1} \beta_{1} & \alpha_{2} & -L_{3} \beta_{3} \cos \theta & 0 \\
0 & L_{3} \beta_{2} \cos \theta & \alpha_{3} & L_{1} \beta_{4} \\
-L_{3} \beta_{1} \cos \theta & 0 & -L_{1} \beta_{3} & \alpha_{4}
\end{array}\right] \\
& \quad \times\left\{\begin{array}{c}
F_{1} \\
F_{2} \\
F_{3} \\
F_{4}
\end{array}\right\} .
\end{aligned}
$$

The subsequent sections discuss the effect of moment excitation on the input power to infinite and finite plates particularly for the multiple point excitation.

\subsection{Infinite Plate Receiver}

6.3.1. Single Contact Point. For a single contact point, Figure 13 shows the input power as a function of $k \alpha$ and the relative phase between the force and moment is not important. However, for multiple contact points, the relative phases are required as the result of the coupling between forces and moments to the response at another contact point.

6.3.2. Multiple Contact Points. As an example, for two contact points there are fifteen relative phases. Assume the distance $L$ between the two points is parallel with $x$-axis $(\delta=0)$ so that some transfer moment mobilities about $x$-axis become zero. The transfer moment mobilities are given in Appendix A. In this case, the total input power for the case of two contact points is given by

$$
\begin{aligned}
P_{\text {in }}= & \frac{1}{2} \operatorname{Re}\left\{\widetilde{Y}_{p}^{v F}\right\}\left(F_{1}^{2}+F_{2}^{2}\right)+\frac{1}{2} \operatorname{Re}\left\{\widetilde{Y}_{p}^{\dot{\theta}_{x} M_{x}}\right\}\left(M_{x, 1}^{2}+M_{x, 2}^{2}\right) \\
& +\frac{1}{2} \operatorname{Re}\left\{\widetilde{Y}_{p}^{\dot{\theta}_{y} M_{y}}\right\}\left(M_{y, 1}^{2}+M_{y, 2}^{2}\right)
\end{aligned}
$$

$$
\begin{aligned}
& +\operatorname{Re}\left\{\tilde{Y}_{t}^{v F}\right\} F_{1} F_{2} \cos \psi_{1}+\operatorname{Re}\left\{\tilde{Y}_{t}^{\dot{\theta}_{x} M_{x}}\right\} M_{x, 1} M_{x, 2} \cos \psi_{2} \\
& +\operatorname{Re}\left\{\tilde{Y}_{t}^{\dot{\theta}_{y} M_{y}}\right\} M_{y, 1} M_{y, 2} \cos \psi_{3} \\
& +\operatorname{Re}\left\{\tilde{Y}_{t}^{v M_{y}}\right\}\left(F_{2} M_{y, 1} \cos \psi_{4}+F_{1} M_{y, 2} \cos \psi_{5}\right)
\end{aligned}
$$

where $Y_{p}$ denotes the point mobility (the same contact point) and $Y_{t}$ denotes the transfer mobility (different contact point). The phase $\psi$ denotes the relative phase between the two components at the same or different contact points; for example, $\psi_{4}$ is the relative phase between the moment about the $y$-axis and the force at different points. In (50), it has been noted that $\widetilde{Y}_{t}^{v M_{y}}=\widetilde{Y}_{t}^{\dot{\theta}_{y} F}$. Due to the complexity of this expression, it is difficult to determine the bounds of input power analytically. However for simplicity, it is assumed that all the components are in-phase, so that $\psi_{i}=0$ for $i=$ $1,2,3,4$, and 5. By also assuming $F_{1}=F_{2}=F, \alpha_{1}=\alpha_{2}$ and following the same method as in (48) and (49) for a $2 \times 2$ matrix, thus, $M_{x, 1}=M_{x, 2}=M_{x}=\alpha F, M_{y, 1}=(\alpha+\beta L) F$, and $M_{y, 2}=(\alpha-\beta L) F$. The asymptotic forms of the transfer mobility in (50) for this case can be expressed as (see also Appendix A)

$$
\begin{aligned}
& \widetilde{Y}_{t}^{v M_{y}}=\frac{2}{k} \operatorname{Re}\left\{\widetilde{Y}_{p}^{\dot{\theta}_{y} M_{y}}\right\} \\
& \times \sqrt{\frac{2}{\pi k L}}\left(\sin \left(k L-\frac{\pi}{4}\right)-j \sin \left(k L-\frac{3 \pi}{4}\right)\right), \\
& \operatorname{Re}\left\{\widetilde{Y}_{t}^{\dot{\theta}_{x} M_{x}}\right\}=\frac{2}{k L} \operatorname{Re}\left\{\widetilde{Y}_{p}^{\dot{\theta}_{x} M_{x}}\right\} \sqrt{\frac{2}{\pi k L}} \sin \left(k L-\frac{\pi}{4}\right), \\
& \operatorname{Re}\left\{\widetilde{Y}_{t}^{\dot{\theta}_{y} M_{y}}\right\}= 2 \operatorname{Re}\left\{\widetilde{Y}_{p}^{\dot{\theta}_{y} M_{y}}\right\} \sqrt{\frac{2}{\pi k L}} \\
& \times\left(\cos \left(k L-\frac{\pi}{4}\right)-\frac{1}{k L} \sin \left(k L-\frac{\pi}{4}\right)\right) .
\end{aligned}
$$

By substituting (51a), (51b), and (51c) into (50) and setting the cos and sin terms equal to unity, the maximum and minimum bounds of the input power normalised with respect to the input power from translational force $\left(P_{F}\right)$ for in-phase excitation are found to be

$$
\begin{aligned}
\frac{\overline{P_{\text {in }}}}{2 P_{F}}=1 & +(k \alpha)^{2}+\frac{(k \beta L)^{2}}{2} \\
& \pm \sqrt{\frac{2}{\pi k L}}\left[1+(k \alpha)^{2}-(k \beta L)^{2}+\frac{(k \beta L)^{2}}{k L}\right] .
\end{aligned}
$$

This reduces to (13), the case where there is only translational force excitation, when $k \alpha \ll 1$ and $k \beta L \ll 1$.

In Section 3.2, assuming random phases with equal probability in (50), the mean and the variance of the input power 


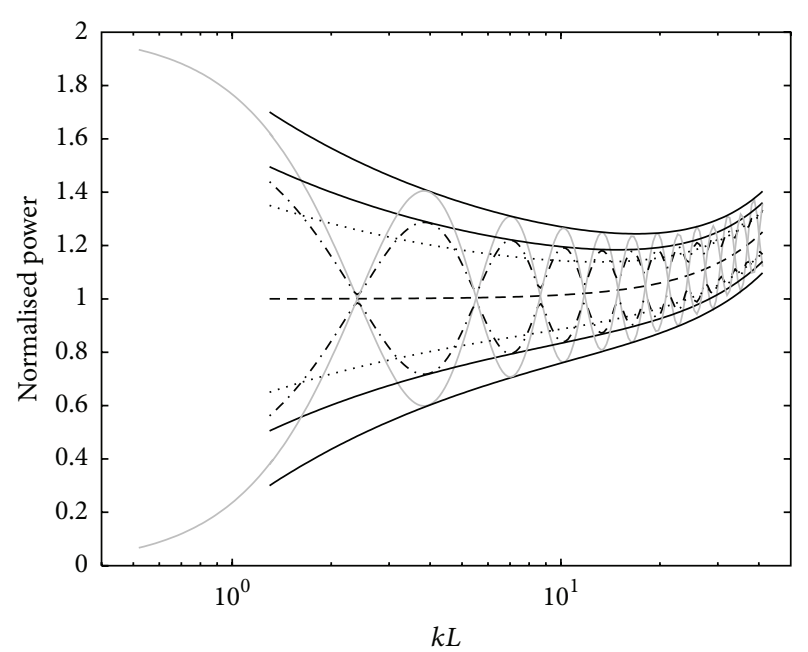

FIGURE 16: The normalised input power to an infinite plate subjected to two harmonic unit point forces and two harmonic moments: - mean, - (thick line) max/min bounds; (52), - - - mean \pm standard deviation and - mean \pm bounds of standard deviation; (55), $\cdots$ mean \pm bounds of standard deviation due to uncertainty in $k L$ and (56) $(\alpha=0.003 \mathrm{~m}, \beta=0.003)$.

to an infinite plate receiver through $N$ contact points can, in general, be expressed as

$$
\begin{aligned}
\mu_{P_{\mathrm{in}}}= & \frac{1}{2} \sum_{i}^{N}\left(\operatorname{Re}\left\{\tilde{Y}_{i i}^{v F}\right\} F_{i}^{2}+\operatorname{Re}\left\{\tilde{Y}_{i i}^{\dot{\theta}_{x, y} M_{x, y}}\right\} M_{x, y(i)}^{2}\right), \\
\sigma_{P_{\mathrm{in}}}^{2}= & \frac{1}{2} \sum_{i=1}^{N-1} \sum_{\substack{k=2 \\
k>i}}^{N}\left(\operatorname{Re}\left\{\tilde{Y}_{i k}^{v F}\right\} F_{i} F_{k}\right)^{2} \\
& +\frac{1}{2} \sum_{i=1}^{N-1} \sum_{\substack{k=2 \\
k>i}}^{N}\left(\operatorname{Re}\left\{\tilde{Y}_{i k}^{\left.\dot{\theta}_{x, y} M_{x, y}\right\}} M_{x, y(i)} M_{x, y(k)}\right)^{2}\right. \\
& +\frac{1}{2} \sum_{i=1}^{N-1} \sum_{\substack{k=2 \\
k>i}}^{N}\left(\operatorname{Re}\left\{\tilde{Y}_{i k}^{\dot{\theta}_{x, y} F}\right\} F_{i} M_{x, y(k)}\right)^{2} \\
& +\frac{1}{2} \sum_{i=1}^{N-1} \sum_{\substack{k=2 \\
k>i}}^{N}\left(\operatorname{Re}\left\{\tilde{Y}_{i k}^{v M_{x, y}}\right\} M_{x, y(i)} F_{k}\right)^{2}
\end{aligned}
$$

where $i$ and $k$ indicate the $i$ th and $k$ th contact points, respectively.

The bounds of the normalised standard deviation can be obtained by substituting (51a), (51b), and (51c) into (54). After algebraic manipulation, it can be approximated by

$$
\begin{aligned}
\frac{\bar{\sigma}}{2 P_{F}} \approx \frac{1}{\sqrt{\pi k L}}[ & 1+\frac{(k \alpha)^{4}}{(k L)^{2}}+\left((k \alpha)^{2}-(k \beta L)^{2}\right)^{2} \\
& \left.+2\left((k \alpha)^{2}+(k \beta L)^{2}\right)\right]^{1 / 2}
\end{aligned}
$$

Again, for $k \alpha \ll 1$ and $k \beta L \ll 1$, this yields the standard deviation for force excitation (see (16)). Following the same method in(17), the standard deviation due to uncertainty in the dimensionless spacing $k L$ is given by

$$
\begin{aligned}
\frac{\bar{\sigma}}{2 P_{F}} \approx \frac{1}{\sqrt{2 \pi k L}}[1 & +\frac{(k \alpha)^{4}}{(k L)^{2}}+\left((k \alpha)^{2}-(k \beta L)^{2}\right)^{2} \\
& \left.+2\left((k \alpha)^{2}+(k \beta L)^{2}\right)\right]^{1 / 2} .
\end{aligned}
$$

Figure 16 shows the mean input power and its standard deviation. The trend is the same as in Figure 2 for translational force excitation, except that the input power tends to increase at high frequencies due to moment excitations.

\subsection{Finite Plate Receiver}

6.4.1. Single Contact Point. For a finite plate, the phase difference between the force and moment becomes important as the cross-mobility is not zero. For the same plate dimensions as in Section 4.1, Figure 17 shows the normalised input power against $k \alpha$ for a single contact point assuming in-phase force and moment. The result in Figure 17(a) shows the increase of the input power due to moment contribution at $k \alpha>$ 0.25 (see also Figure 13). For the case where the excitation is near to the plate edge in Figure 17(b), the total power is significantly less at low $k \alpha$, because the point mobility for force excitation (which dominates at low frequencies, $k \alpha \ll$ 1 ) is smaller near the edge. However, when $k \alpha>0.2$, the input power is the same as that when the excitation position is near to the centre of the plate due to the increasing power from the moment, so that it compensates partly for the reducing power from the force.

Figure 18 shows the normalised input power for various forcing locations on the plate. The increase in the mean power due to the contribution of moment excitation can be seen roughly above $k \alpha>0.35$.

Figure 19 shows the relative standard deviation $r_{\sigma}$ of the averaged input power for different damping loss factors and magnitudes of moment excitation. For all cases, it can be seen that the relative standard deviation, in an average sense, agrees reasonably well with that from the translational force from (33). This indicates that the ratio between the mean and standard deviation is approximately the same even if the moment excitation is neglected in the calculation of the input power. Particular attention is focused on the results at large $k \alpha$ when the moment starts to contribute substantially to the total input power.

6.4.2. Multiple Contact Points. Again, the relative phases due to coupling between forces and moments are of interest for multiple contact points. The mean power, assuming the relative phases between the excitations are equally probable, is the same as that in (53) for an infinite plate. However, for a finite plate a force will produce a rotation and a moment 


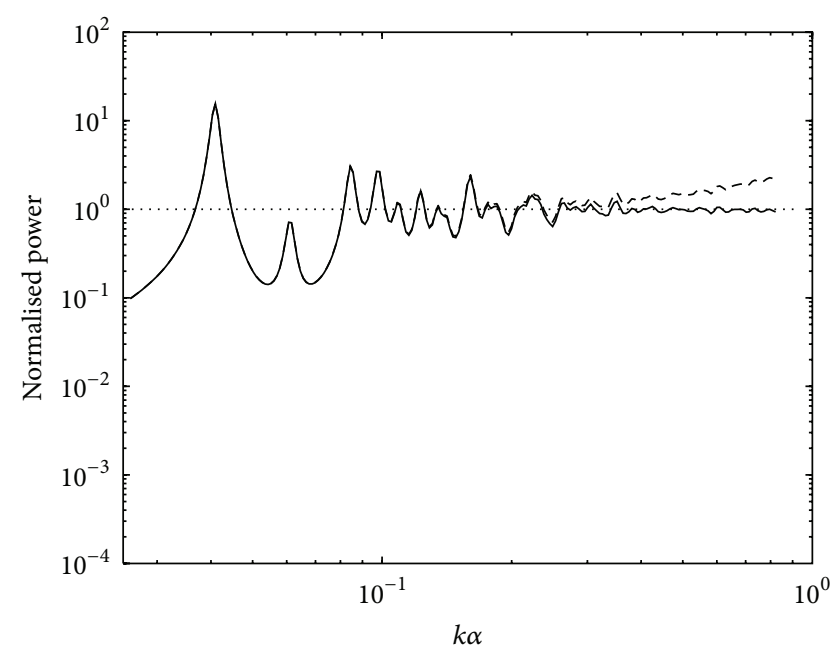

(a)

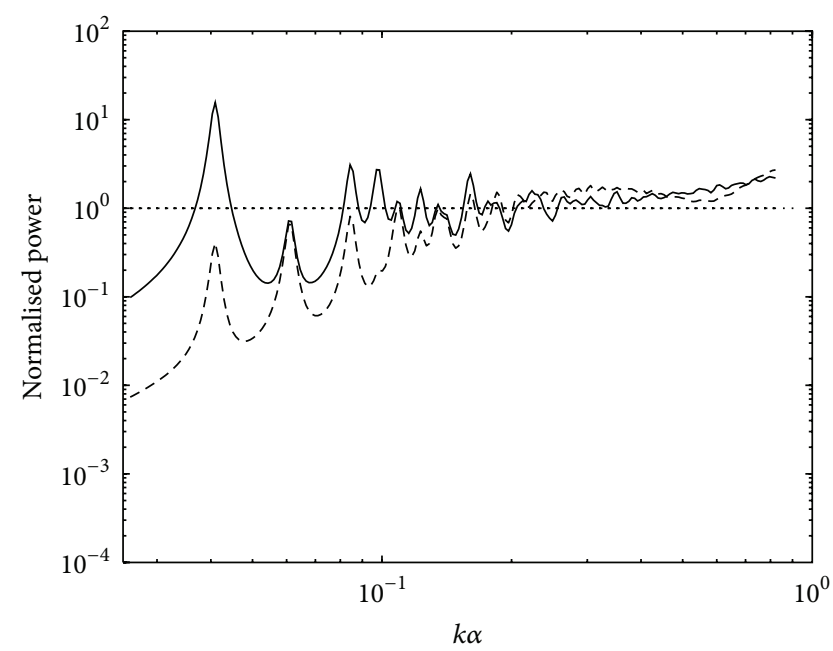

(b)

FIGURE 17: The normalised input power of a finite plate subjected to force and moment excitations at a single contact point ((a) the power with $(--)$ and without $(-)$ moment and (b) the total power for the contact point around the edge $(--)$ and middle $(-)$ of the plate: $\alpha=0.005 \mathrm{~m}$, $\eta=0.1)$.

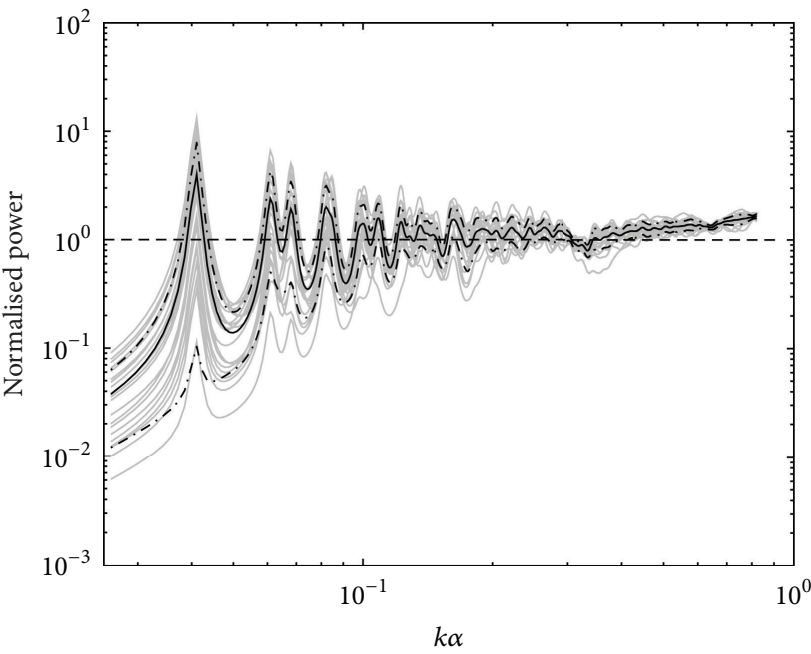

(a)

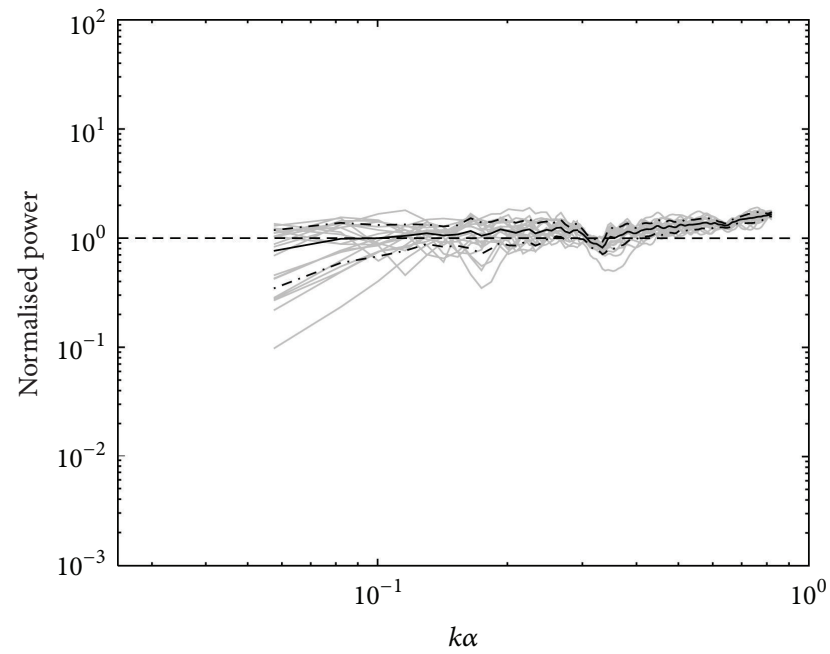

(b)

FIGURE 18: The normalised input power to a finite plate subjected to force and moment excitations at single contact point for various possible forcing locations (grey lines): - mean and $-\cdot-$ mean \pm standard deviation. (a) discrete frequency and (b) frequency-band average; $\alpha=$ $0.005 \mathrm{~m}, \eta=0.1$.

will produce a displacement at the same point. Therefore, the variance is given by

$$
\begin{aligned}
\sigma_{P_{\mathrm{in}}}^{2}= & \frac{1}{2} \sum_{i=1}^{N-1} \sum_{\substack{k=2 \\
k>i}}^{N}\left(\operatorname{Re}\left\{\widetilde{Y}_{v F}^{(i k)}\right\} F_{i} F_{k}\right)^{2} \\
& +\frac{1}{2} \sum_{i=1}^{N-1} \sum_{\substack{k=2 \\
k>i}}^{N}\left(\operatorname{Re}\left\{\widetilde{Y}_{\dot{\theta}_{x, y}^{(i k)}} M_{x, y}\right\} M_{x, y(i)} M_{x, y(k)}\right)^{2}
\end{aligned}
$$

$$
\begin{aligned}
& +\frac{1}{2} \sum_{i=1}^{N} \sum_{\substack{k=1 \\
k \geq i}}^{N}\left(\operatorname{Re}\left\{\widetilde{Y}_{\dot{\theta}_{x, y} F}^{(i k)}\right\} F_{i} M_{x, y(k)}\right)^{2} \\
& +\frac{1}{2} \sum_{i=1}^{N} \sum_{\substack{k=1 \\
k \geq i}}^{N}\left(\operatorname{Re}\left\{\widetilde{Y}_{v M_{x, y}}^{(i k)}\right\} M_{x, y(i)} F_{k}\right)^{2} .
\end{aligned}
$$

For four contact points, the mobility matrices are $12 \times$ 12. Using (53) and (57), Figure 20(a) shows the mean and standard deviation of the input power for damping loss factor 


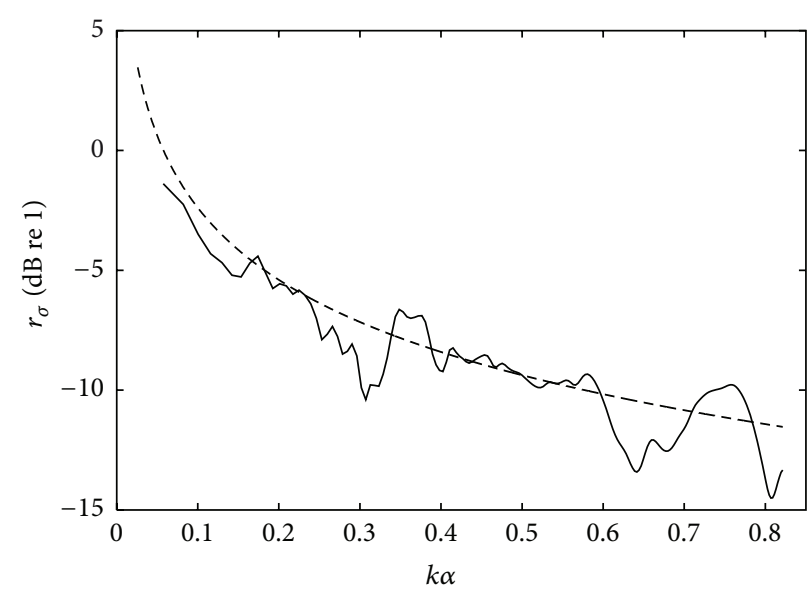

(a)

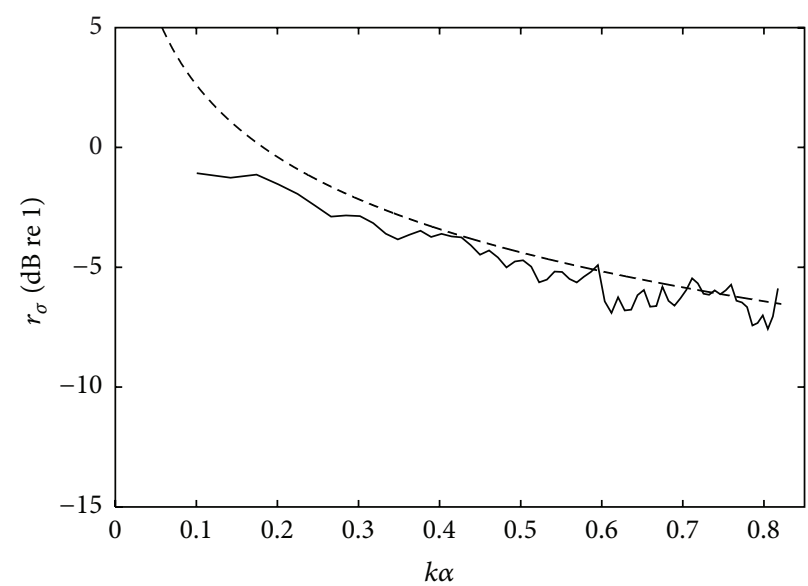

(b)

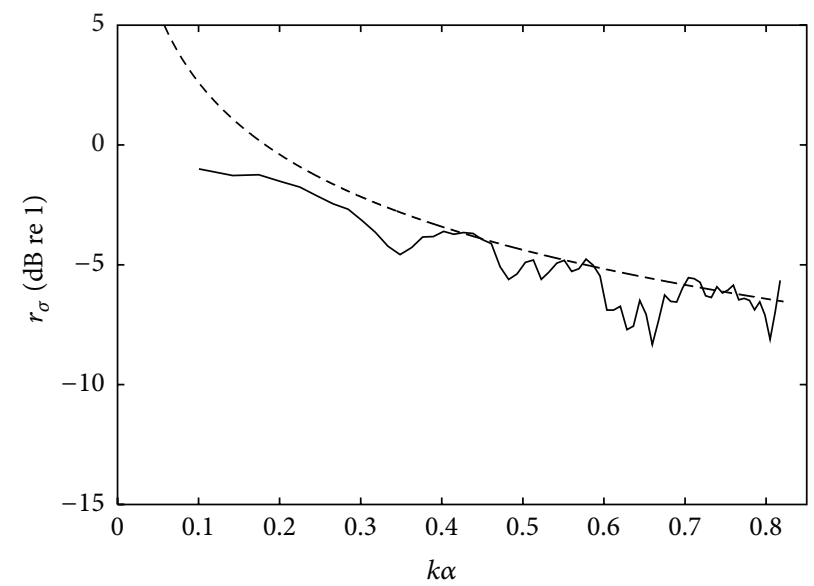

(c)

FIGURE 19: The relative standard deviation of the input power to a finite plate subjected to force and moment excitations at single contact point averaged over various possible forcing locations and frequency bands; - numerical and - - (33) ((a) $\alpha=0.005 \mathrm{~m}, \eta=0.1$; (b) $\alpha=0.005 \mathrm{~m}$, $\eta=0.01 ;$ and (c) $\alpha=0.01 \mathrm{~m}, \eta=0.01$ ).

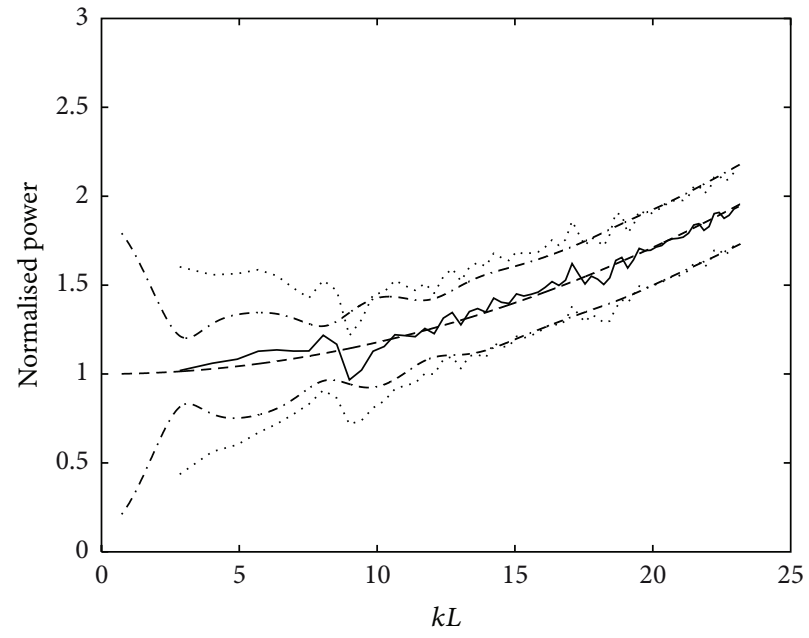

(a)

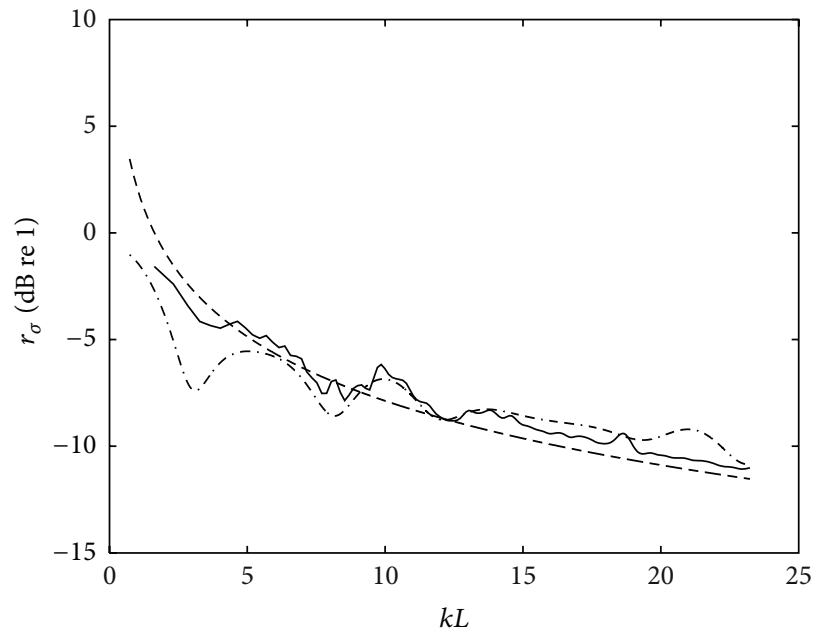

(b)

FiguRE 20: (a) The normalised input power to a finite plate subjected to force and moment excitations at four contact points averaged over various possible forcing locations and frequency bands: mean (- numerical calculation, - - infinite plate) and mean \pm standard deviation $(\cdots$ numerical calculation, $-\cdot-$ infinite plate). (b) The relative standard deviation of the input power; - numerical calculation, $-\cdot-$ infinite plate, and $--(33)(L=0.14 \mathrm{~m}, \eta=0.05, \alpha=0.005 \mathrm{~m}$, and $\beta=0.005)$. 


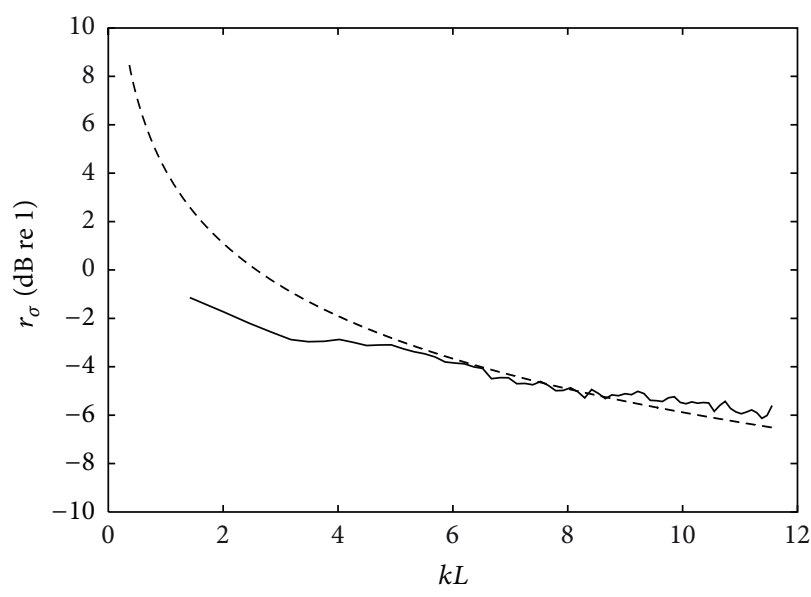

(a)

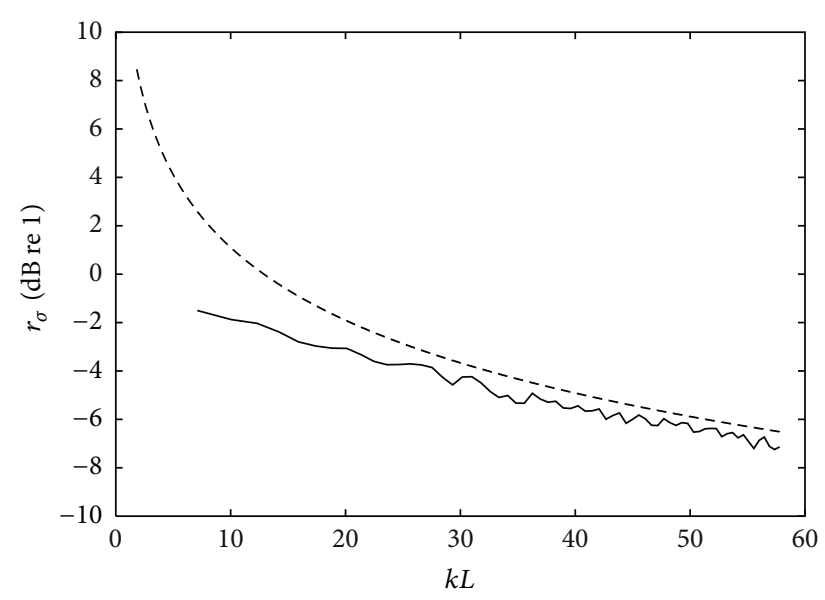

(b)

FIGURE 21: The relative standard deviation of the input power to a finite plate subjected to force and moment excitations at four contact points averaged over various possible forcing locations and frequency bands; - numerical calculation and $--(33)(\eta=0.01, \alpha=0.005 \mathrm{~m}$, and $\beta=0.005$; (a) $L=0.07 \mathrm{~m}$ and (b) $L=0.35 \mathrm{~m}$ ).

$\eta=0.05$. The spatial separation of the contact points is again assumed to form a rectangular shape and $L$ is the length of the diagonal. The results agree well with those from the infinite plate above $k L=10$. Below this, as in Section 4.4 , the agreement deteriorates due to small damping. This is clearly shown in the relative standard deviation plotted in Figure 20(b). However, it can be seen that the numerical result has a good agreement with that from the prediction using (33).

Figure 21 shows the relative standard deviation for $\eta=$ 0.01 for different distances between the contact points. Again, good agreement can be seen using (33), particularly for high frequencies. In Figure 21(b) differences are seen for $k L>25$, but they are less than $1 \mathrm{~dB}$. At low $k L$, the prediction differs by $2 \mathrm{~dB}$ on average due to the very low-modal overlap. From the results presented, it shows that (33), which is applicable only for the translational force, can also be used to predict the contribution of moment at high frequencies.

\section{Conclusions}

The uncertainty in input power to a structure due to uncertainty in the excitation has been investigated. For an infinite plate, the distance between the location where multiple forces are applied is not important if it is less than half a structural wavelength. The variance of the input power due to uncertainty in excitation phase and location tends to decrease as the nondimensional frequency $k L$ increases. For multiple point excitation where the relative phases are random, the mean power and the variance depend only on the input and transfer mobility, respectively.

As for the infinite plate, the variance of the input power to a finite plate also typically decreases as the frequency increases. The frequency average of the input power over all possible forcing locations from multiple contact points can be estimated reasonably and accurately by using the infinite plate result. However, for a very low damping $(<5 \%)$ the agreement deteriorates and the simple prediction of the mean and variance can be used $[1,17]$.

The uncertainty in the force amplitude at the contact points has also been discussed. Unless the spatial separation of the excitation locations is small, the distribution of the force amplitude through the contact points is important to obtain accurate estimates of the variation of the mean and standard deviation of the input power, particularly at low frequencies. This variation reduces as the damping loss factor is increased.

The relative effect of moment excitation can be expressed in terms of a force and a distance corresponding to a characteristic of the source. It can also be scaled as a function of the input power of the force and the structural wavenumber. This effect tends to increase as frequency increases. The contribution to the total input power can be predicted using the simple expression of the relative standard deviation for the force. However in any event, the effects of moment excitation are typically small at low frequency and in any event are generally less than the effects of force excitation. They are typically, thus, of secondary importance.

Finally, there remains the moot point of what uncertainty is, in practice, acceptable. This is to a large extent dependent on the typical uncertainty of machinery characterisation methods, such as the reception plate method. The attempt here is to quantify to some extent the uncertainty introduced by some details of the excitation, details that would typically not be measured.

\section{Appendices}

\section{A. Force and Moment Transfer Mobilities for an Infinite plate}

Figure 14 defines the force-moment excitation directions. The same directions are also applied to the response at another 
point at distance $L$ away from the excitation point. The $z$-axis is perpendicular to the surface of the plate. The mobility terms of an infinite plate structure subjected to a harmonic force or moment point loading are given by [15]

$$
\begin{aligned}
& \tilde{Y}^{v F}=\frac{\omega}{8 B k^{2}}\left[H_{0}^{(2)}(k L)-\frac{2 j}{\pi} K_{0}(k L)\right], \\
& \tilde{Y}^{v M_{x}}=\tilde{Y}^{\dot{\theta}_{x} F}=\frac{\omega \sin \delta}{8 B k}\left[H_{1}^{(2)}(k L)-\frac{2 j}{\pi} K_{1}(k L)\right] \text {, } \\
& \tilde{Y}^{v M_{y}}=\tilde{Y}^{\dot{\theta}_{y} F}=\frac{\omega \cos \delta}{8 B k}\left[H_{1}^{(2)}(k L)-\frac{2 j}{\pi} K_{1}(k L)\right] \text {, } \\
& \tilde{Y}^{\dot{\theta}_{x} M_{x}}=\frac{\omega}{8 B}\left[\sin ^{2} \delta\left(H_{0}^{(2)}(k L)+\frac{2 j}{\pi} K_{0}(k L)\right)\right. \\
& +\frac{1}{k L}\left(\cos ^{2} \delta-\sin ^{2} \delta\right) \\
& \left.\times\left(H_{1}^{(2)}(k L)-\frac{2 j}{\pi} K_{1}(k L)\right)\right], \\
& \tilde{Y}^{\dot{\theta}_{y} M_{y}}=\frac{\omega}{8 B}\left[\cos ^{2} \delta\left(H_{0}^{(2)}(k L)+\frac{2 j}{\pi} K_{0}(k L)\right)\right. \\
& +\frac{1}{k L}\left(\sin ^{2} \delta-\cos ^{2} \delta\right) \\
& \left.\times\left(H_{1}^{(2)}(k L)-\frac{2 j}{\pi} K_{1}(k L)\right)\right], \\
& \tilde{Y}^{\dot{\theta}_{y} M_{x}}=\widetilde{Y}_{M_{y} \dot{\theta}_{x}} \\
& =\frac{\omega}{8 B}\left[-\sin \delta \cos \delta\left(H_{0}^{(2)}(k L)+\frac{2 j}{\pi} K_{0}(k L)\right)\right. \\
& \left.+\frac{2}{k L} \cos \delta \sin \delta\left(H_{1}^{(2)}(k L)-\frac{2 j}{\pi} K_{1}(k L)\right)\right],
\end{aligned}
$$

where $B$ is the bending stiffness, $k$ is the structural wavenumber, $H_{n}^{(2)}$ is the $n$ th-order Hankel function of the second kind, and $K_{n}$ is the $n$ th-order modified Bessel function of the second kind. The asymptotic forms of the functions for $k L \gg 1$ are given by [16]

$$
\begin{gathered}
H_{n}^{(2)} \approx \sqrt{\frac{2}{\pi k L} e^{-j(k L-(1 / 2) n \pi-(1 / 4) \pi)},} \\
K_{n}(k L) \approx \sqrt{\frac{\pi}{2 k L}} e^{-k L} \\
\times\left[1+\frac{\epsilon-1}{8 k L}+\frac{(\epsilon-1)(\epsilon-9)}{2 !(8 k L)^{2}}\right. \\
\left.+\frac{(\epsilon-1)(\epsilon-9)(\epsilon-25)}{3 !(8 k L)^{3}}+\cdots\right],
\end{gathered}
$$

where $\epsilon=4 n$. For very large $k L, K n(k L) \rightarrow 0$.

\section{B. Force and Moment Mobilities for a Finite Plate}

For a finite rectangular plate, the mobilities can be written in terms of a modal summation. The point reference $(0,0)$ is located at the corner of the plate. The moment-rotational velocity transfer mobilities at frequency $\omega$ for a plate with damping loss factor $\eta$ are given by [15]

$$
\begin{aligned}
& \tilde{Y}^{\dot{\theta}_{x} M_{x}}=j \omega \sum_{p=1}^{\infty} \sum_{q=1}^{\infty} \frac{(\partial / \partial y)\left[\Phi_{p q}\left(x_{0}, y_{0}\right)\right](\partial / \partial y)\left[\Phi_{p q}(x, y)\right]}{\omega_{p q}^{2}(1+j \eta)-\omega^{2}}, \\
& \tilde{Y}^{\dot{\theta}_{y} M_{y}}=j \omega \sum_{p=1}^{\infty} \sum_{q=1}^{\infty} \frac{(\partial / \partial x)\left[\Phi_{p q}\left(x_{0}, y_{0}\right)\right](\partial / \partial x)\left[\Phi_{p q}(x, y)\right]}{\omega_{p q}^{2}(1+j \eta)-\omega^{2}}, \\
& \tilde{Y}^{\dot{\theta}_{y} M_{x}}=-j \omega \sum_{p=1}^{\infty} \sum_{q=1}^{\infty} \frac{(\partial / \partial y)\left[\Phi_{p q}\left(x_{0}, y_{0}\right)\right](\partial / \partial x)\left[\Phi_{p q}(x, y)\right]}{\omega_{p q}^{2}(1+j \eta)-\omega^{2}}, \\
& \tilde{Y}^{\dot{\theta}_{x} M_{y}}=-j \omega \sum_{p=1}^{\infty} \sum_{q=1}^{\infty} \frac{(\partial / \partial x)\left[\Phi_{p q}\left(x_{0}, y_{0}\right)\right](\partial / \partial y)\left[\Phi_{p q}(x, y)\right]}{\omega_{p q}^{2}(1+j \eta)-\omega^{2}}
\end{aligned}
$$

and the force-rotational and the moment-translational velocity transfer mobilities are

$$
\begin{gathered}
\tilde{Y}^{\dot{\theta}_{x} F}=j \omega \sum_{p=1}^{\infty} \sum_{q=1}^{\infty} \frac{\Phi_{p q}\left(x_{0}, y_{0}\right)(\partial / \partial y)\left[\Phi_{p q}(x, y)\right]}{\omega_{p q}^{2}(1+j \eta)-\omega^{2}} \\
\tilde{Y}^{\dot{\theta}_{y} F}=-j \omega \sum_{p=1}^{\infty} \sum_{q=1}^{\infty} \frac{\Phi_{p q}\left(x_{0}, y_{0}\right)(\partial / \partial x)\left[\Phi_{p q}(x, y)\right]}{\omega_{p q}^{2}(1+j \eta)-\omega^{2}}, \\
\tilde{Y}^{v M_{x}}=j \omega \sum_{p=1}^{\infty} \sum_{q=1}^{\infty} \frac{(\partial / \partial y)\left[\Phi_{p q}\left(x_{0}, y_{0}\right)\right] \Phi_{p q}(x, y)}{\omega_{p q}^{2}(1+j \eta)-\omega^{2}} \\
\tilde{Y}^{v M_{y}}=-j \omega \sum_{p=1}^{\infty} \sum_{q=1}^{\infty} \frac{(\partial / \partial x)\left[\Phi_{p q}\left(x_{0}, y_{0}\right)\right] \Phi_{p q}(x, y)}{\omega_{p q}^{2}(1+j \eta)-\omega^{2}}
\end{gathered}
$$

where $\Phi_{p q}$ is the mass normalised mode shape and $\omega_{p q}$ is the natural frequency of the $(p, q)$ mode as defined in (28) for a simply supported boundary condition. The point mobility can be obtained by setting $x=x_{0}$ and $y=y_{0}$.

\section{Acknowledgments}

The authors gratefully acknowledge the financial support provided by the Engineering and Physical Sciences Research Council (EPSRC) under Grant EP/D002 15X/1. The authors also acknowledge the valuable discussions with Professor $B$. M. Gibbs from the University of Liverpool and Dr. A. T. Moorhouse from the University of Salford. 


\section{References}

[1] R. S. Langley and A. W. M. Brown, "The ensemble statistics of the energy of a random system subjected to harmonic excitation," Journal of Sound and Vibration, vol. 275, no. 3-5, pp. 823846, 2004.

[2] R. S. Langley and A. W. M. Brown, "The ensemble statistics of the band-averaged energy of a random system," Journal of Sound and Vibration, vol. 275, no. 3-5, pp. 847-857, 2004.

[3] R. S. Langley and V. Cotoni, "Response variance prediction in the statistical energy analysis of built-up systems," Journal of the Acoustical Society of America, vol. 115, no. 2, pp. 706-718, 2004.

[4] J. M. Mondot and B. Petersson, "Characterization of structureborne sound sources: the source descriptor and the coupling function," Journal of Sound and Vibration, vol. 114, no. 3, pp. 507-518, 1987.

[5] B. A. T. Petersson and B. M. Gibbs, "Use of the source descriptor concept in studies of multi-point and multi-directional vibrational sources," Journal of Sound and Vibration, vol. 168, no. 1, pp. 157-176, 1993.

[6] B. Petersson and J. Plunt, "On effective mobilities in the prediction of structure-borne sound transmission between a source structure and a receiving structure, part I: theoretical background and basic experimental studies," Journal of Sound and Vibration, vol. 82, no. 4, pp. 517-529, 1982.

[7] B. Petersson and J. Plunt, "On effective mobilities in the prediction of structure-borne sound transmission between a source structure and a receiving structure, part II: procedures for the estimation of mobilities," Journal of Sound and Vibration, vol. 82, no. 4, pp. 531-540, 1982.

[8] R. A. Fulford and B. M. Gibbs, "Structure-borne sound power and source characterisation in multi-point-connected systems, Part 1: case studies for assumed force distributions," Journal of Sound and Vibration, vol. 204, no. 4, pp. 659-677, 1997.

[9] R. A. Fulford and B. M. Gibbs, "Structure-borne sound power and source characterization in multi-point-connected systems. Part 3: force ratio estimates," Journal of Sound and Vibration, vol. 225, no. 2, pp. 239-282, 1999.

[10] L. Cremer, M. Heckl, and B. A. T. Petersson, Structure Borne Sound, Springer, Berlin, Germany, 3rd edition, 2005.

[11] B. M. Gibbs, R. Cookson, and N. Qi, "Vibration activity and mobility of structure-borne sound sources by a reception plate method," Journal of the Acoustical Society of America, vol. 123, no. 6, pp. 4199-4209, 2008.

[12] B. A. T. Petersson and B. M. Gibbs, "Towards a structure-borne sound source characterization," Applied Acoustics, vol. 61, no. 3, pp. 325-343, 2000.

[13] T. A. Evans and A. T. Moorhouse, "Mean and variance of injected structure borne sound power due to missing source activity phase data," Proceeding of NOVEM, Oxford, UK, 2009.

[14] L. Hinke, Modelling approaches for the low-frequency analysis of the built-up structures with non-deterministic properties [Ph.D. thesis], University of Southampton, 2008.

[15] M. J. Brennan and P. Gardonio, "Mobility and impedance methods in structural dynamics," in Chapter 9 in Advanced Applications in Acoustics, Noise and Vibration, F. J. Fahy and J. G. Walker, Eds., Spon Press, 2004.

[16] M. Danos and J. Rafelski, Pocketbook of Mathematical Functions, Harri Deutsch; Abridged, 1984.

[17] R. H. Lyon and R. G. DeJong, Application of Statistical Energy Analysis, Butterworth-Heinemann, 1995.
[18] A. T. Moorhouse, "A dimensionless mobility formulation for evaluation of force and moment excitation of structures," Journal of the Acoustical Society of America, vol. 112, no. 3, pp. 972980, 2002.

[19] S. H. Yap and B. M. Gibbs, "Structure-borne sound transmission from machines in buildings, part 2: indirect measurement of force and moment at the machine-receiver interface of a single point connected system by a reciprocal method," Journal of Sound and Vibration, vol. 222, no. 1, pp. 99-113, 1999.

[20] B. A. T. Petersson, "Structural acoustic power transmission by point moment and force excitation, Part II: plate-like structures," Journal of Sound and Vibration, vol. 160, no. 1, pp. 67-91, 1993. 

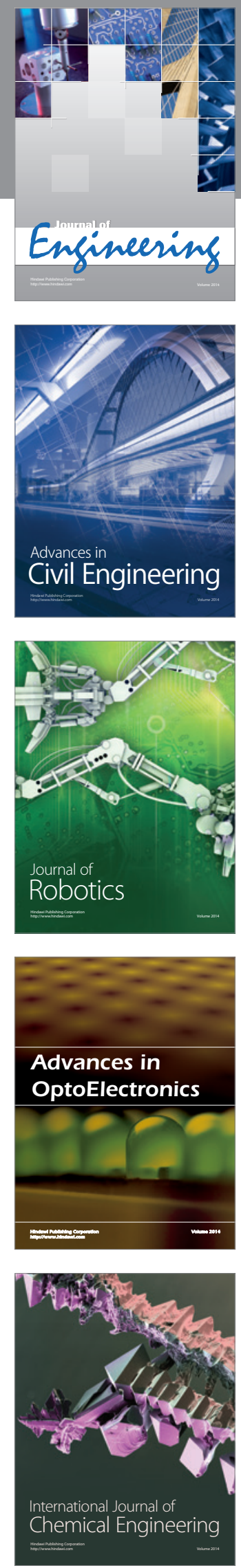

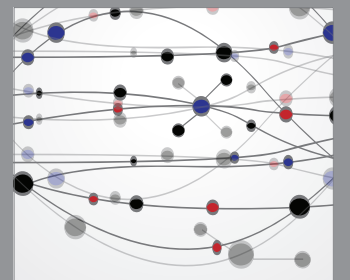

The Scientific World Journal
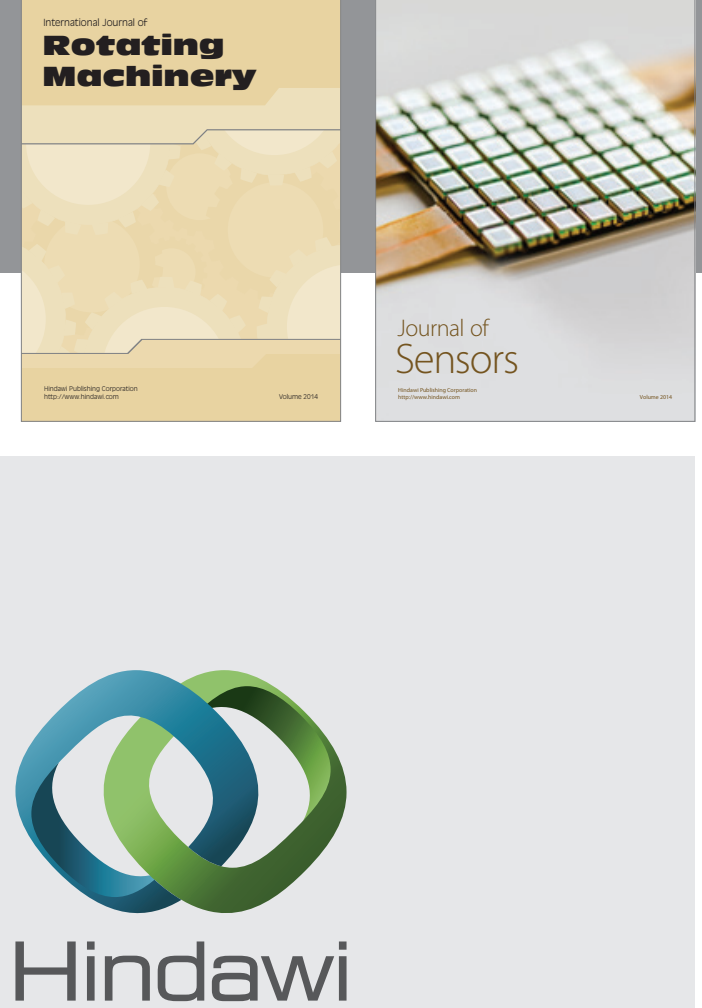

Submit your manuscripts at http://www.hindawi.com
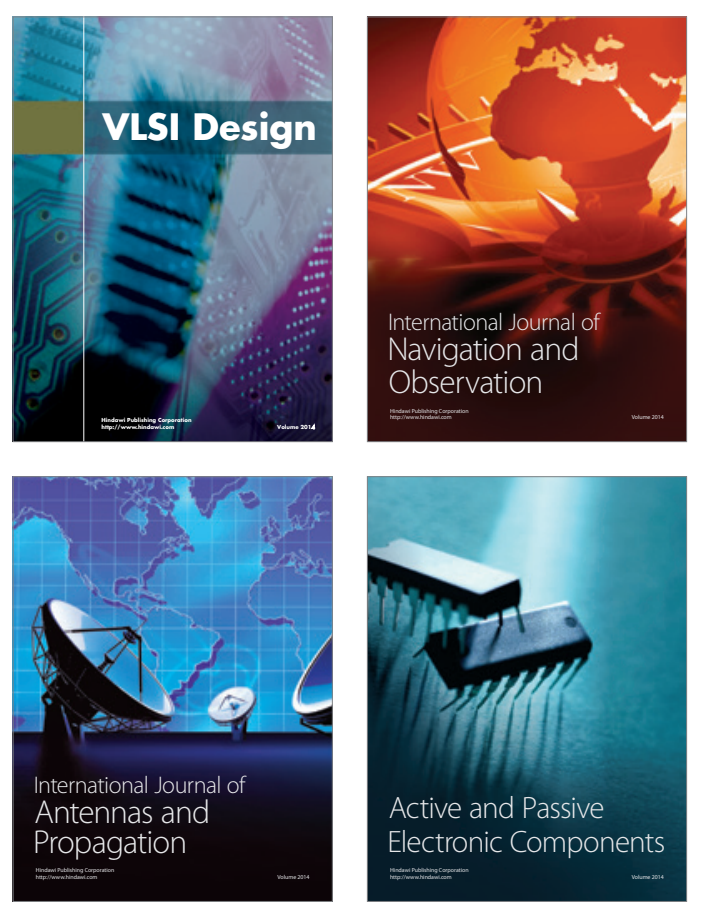
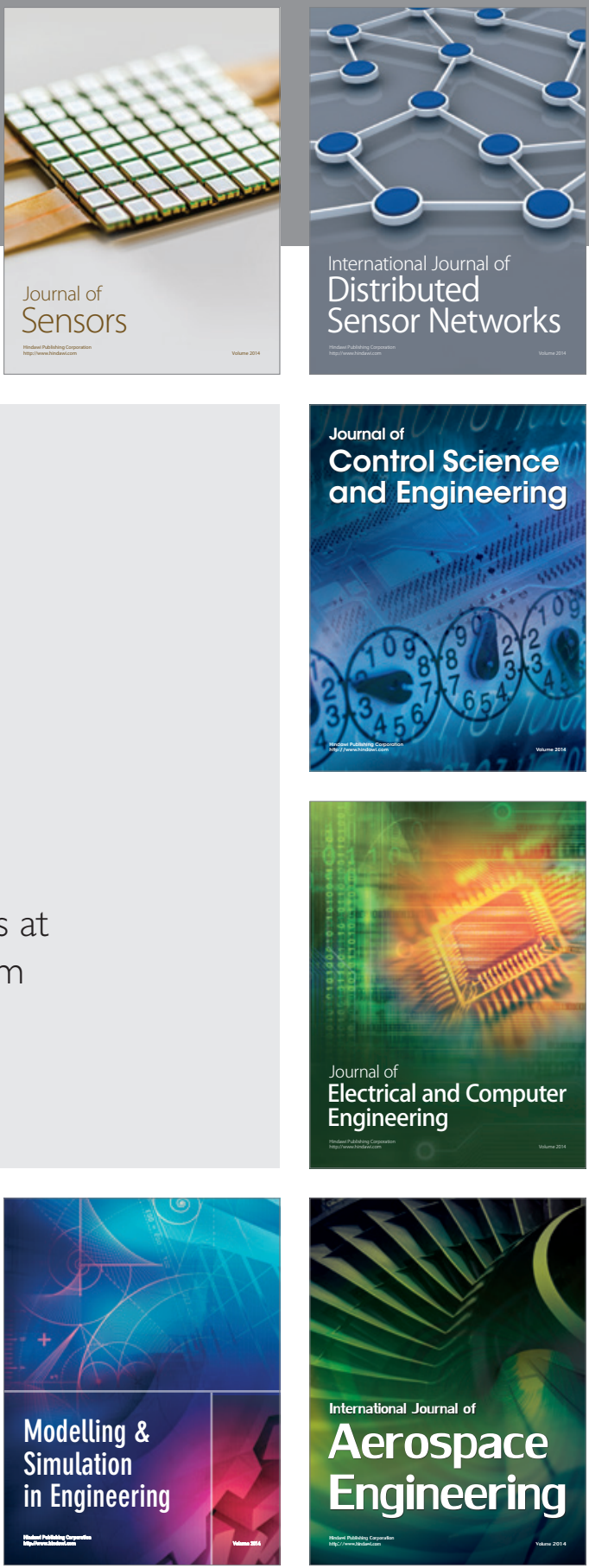

Journal of

Control Science

and Engineering
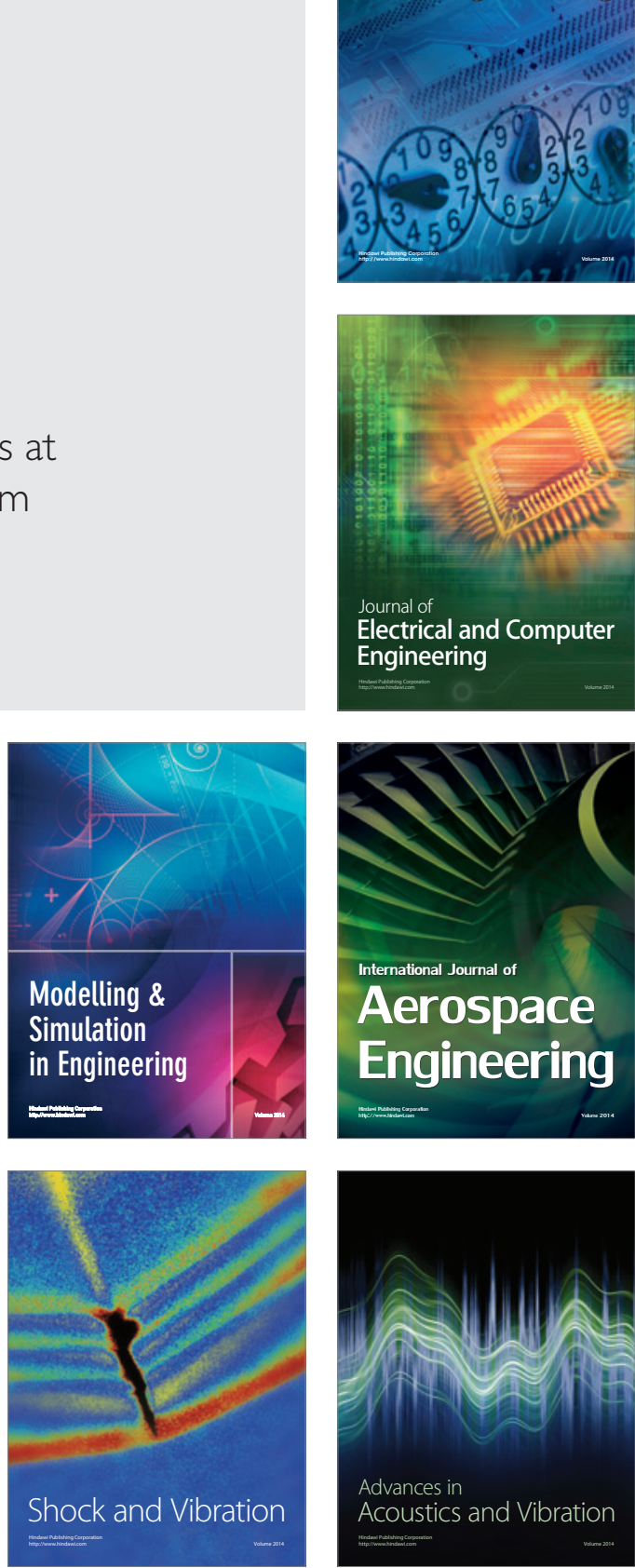